Revue québécoise de droit international

Quebec Journal of International Law

Revista quebequense de derecho internacional

\title{
Les dimensions et les dynamiques régionales et internationales de la justice transitionnelle au Burundi : proposition de création d'un organe de droit pénal international au sein de la Communauté Est-Africaine
}

\section{Nestor Nkurunziza}

Numéro hors-série, décembre 2017

Études de certains grands enjeux de la justice internationale pénale

URI : https://id.erudit.org/iderudit/1056229ar

DOI : https://doi.org/10.7202/1056229ar

Aller au sommaire du numéro

Éditeur(s)

Société québécoise de droit international

ISSN

0828-9999 (imprimé)

2561-6994 (numérique)

Découvrir la revue

Citer cet article

Nkurunziza, N. (2017). Les dimensions et les dynamiques régionales et internationales de la justice transitionnelle au Burundi : proposition de création d'un organe de droit pénal international au sein de la Communauté Est-Africaine. Revue québécoise de droit international / Quebec Journal of International Law / Revista quebequense de derecho internacional, 167-203. https://doi.org/10.7202/1056229ar
Résumé de l'article

Cet article utilise le Burundi comme principal cas d'étude pour proposer la création d'un organe de droit pénal international au sein de la Communauté des États d'Afrique de l'Est (EAC). L'article tient compte des projets en cours au sein de l’Union Africaine vers une régionalisation de la justice pénale internationale en Afrique. Il repose en outre sur une pratique prometteuse de l'actuelle Cour de Justice de l'EAC (EACJ) dans les domaines de la bonne gouvernance, l'État de droit et les droits de l'homme. Même si le Burundi est utilisé comme principal cas d'étude, les arguments avancés valent également dans les contextes de la plupart des États membres de la Communauté en situation de transition post conflit, avec des défis comparables en matière d'État de droit. L'argument principal de l'article repose sur l'idée qu'une mise en oeuvre régionaliste du droit pénal international offre des avantages uniques ainsi qu'un potentiel plus élevé pour atteindre des objectifs d'une importance capitale dans les contextes post conflit d'administration de la justice pénale internationale. Par conséquent, l'auteur propose qu'en cas de conflits de compétence entre des juridictions toutes supra-nationales (sous-régional, continental, international), envisageables dorénavant, la juridiction internationale ayant la plus grande proximité avec le territoire du pays où les crimes ont été commis jouit d'une priorité d'intervention, à défaut de poursuites nationales. Situant cet argument dans le contexte de la crise que connait la Cour Pénale Internationale (CPI) sur le continent africain, l'auteur soutient qu'en théorie cette solution qui revient à promouvoir les juridictions régionales comme sphères privilégiées d'application du droit pénal international ne s'inscrit pas nécessairement en porte à faux avec une bonne coopération en matière d'intervention pénale internationale. Au contraire, cette solution est règle générale mieux conforme aux fondements et aux buts d'une politique de complémentarité positive en matière d'intervention pénale internationale dans le contexte d'une pluralité de juridictions supra-nationales de droit pénal international. 


\title{
LES DIMENSIONS ET LES DYNAMIQUES RÉGIONALES ET INTERNATIONALES DE LA JUSTICE TRANSITIONNELLE AU BURUNDI : PROPOSITION DE CRÉATION D'UN ORGANE DE DROIT PÉNAL INTERNATIONAL AU SEIN DE LA COMMUNAUTÉ EST-AFRICAINE
}

\author{
Nestor Nkurunziza*
}

\begin{abstract}
Cet article utilise le Burundi comme principal cas d'étude pour proposer la création d'un organe de droit pénal international au sein de la Communauté des États d'Afrique de l'Est (EAC). L'article tient compte des projets en cours au sein de l'Union Africaine vers une régionalisation de la justice pénale internationale en Afrique. Il repose en outre sur une pratique prometteuse de l'actuelle Cour de Justice de l'EAC (EACJ) dans les domaines de la bonne gouvernance, l'État de droit et les droits de l'homme. Même si le Burundi est utilisé comme principal cas d'étude, les arguments avancés valent également dans les contextes de la plupart des États membres de la Communauté en situation de transition post conflit, avec des défis comparables en matière d'État de droit. L'argument principal de l'article repose sur l'idée qu'une mise en œuvre régionaliste du droit pénal international offre des avantages uniques ainsi qu'un potentiel plus élevé pour atteindre des objectifs d'une importance capitale dans les contextes post conflit d'administration de la justice pénale internationale. Par conséquent, l'auteur propose qu'en cas de conflits de compétence entre des juridictions toutes supra-nationales (sous-régional, continental, international), envisageables dorénavant, la juridiction internationale ayant la plus grande proximité avec le territoire du pays où les crimes ont été commis jouit d'une priorité d'intervention, à défaut de poursuites nationales. Situant cet argument dans le contexte de la crise que connait la Cour Pénale Internationale (CPI) sur le continent africain, l'auteur soutient qu'en théorie cette solution qui revient à promouvoir les juridictions régionales comme sphères privilégiées d'application du droit pénal international ne s'inscrit pas nécessairement en porte à faux avec une bonne coopération en matière d'intervention pénale internationale. $\mathrm{Au}$ contraire, cette solution est règle générale mieux conforme aux fondements et aux buts d'une politique de complémentarité positive en matière d'intervention pénale internationale dans le contexte d'une pluralité de juridictions supra-nationales de droit pénal international.
\end{abstract}

This paper uses Burundi as its main case study to suggest the creation of an international criminal justice mechanism within the current institutional structures of the East-African Community (EAC).The contribution takes stock of ongoing developments within the African Union towards a regionalization of international criminal justice in Africa. It also draws some of its arguments from a promising practice of the current East-African Court of Justice (EACJ) as an analysis of the court's case-law in the fields related to the rule of law, governance and human rights shows. The main argument of the article is that trials have a greater potential to achieve some important goals of transitional justice and international criminal justice if they are undertaken by a court with a physical proximity to the areas where crimes were committed, rather than at a global level. Although Burundi is used as the main case study, it is believed that such a mechanism would bring a contribution in the promotion of the rule of law in most of country members of the EAC facing similar rule of law challenges in post-conflict settings. Although the paper highlights the advantages of prosecutions and trials carried out on a regional basis, the author recognizes the necessity for cooperation among national and supra-national actors involved in fighting the impunity of serious crimes, particularly in Africa. For the purpose of a positive complementarity in the administration of international criminal justice, it is suggested that a better approach to an international intervention should recognize geographic proximity as the primary criteria to determine which supra-national judicial institution (subregional, continental, global) should have the priority to intervene in the absence of opportunities for justice at the national level. Such solution would better comply with the objectives and rationale of a positive approach to complementarity in this context.

\footnotetext{
* Candidat au doctorat en droit, Université d’Ottawa.
} 
Este artículo propone la creación de un cuerpo de derecho penal internacional en la Comunidad del África Oriental (EAC), para esto utiliza como principal caso de estudio a Burundi. El artículo tiene en cuenta los proyectos en curso dentro de la Unión Africana hacia una regionalización de la justicia penal internacional en África. Así mismo, considera la jurisprudencia del Tribunal de Justicia actual de la EAC (EACJ) en las áreas de buen gobierno, el estado de derecho y los derechos humanos. El principal argumento es desarrollado con base a la idea de que una aplicación regionalista del derecho penal internacional ofrece una mejor solución para cumplir los principales objetivos de la justicia penal internacional en contextos de post-conflicto, los cuales son difíciles de cumplir en un contexto global. Aunque Burundi se utiliza como caso de estudio principal, los argumentos pueden también ser aplicados a los contextos de post-conflicto de la mayor parte de los Estados miembros de la Comunidad. El autor sugiere que en el caso de un conflicto de competencia entre los tribunales supranacionales (sub regional, continental, internacional), el tribunal internacional con mayor proximidad al territorio del país donde se cometieron los crímenes tienen prioridad de jurisdicción. Esta solución satisface mejor las bases de una política de complementariedad positiva en la administración de la justicia penal internacional en ese contexto. 
L'évolution historique de la justice pénale internationale montre que les juridictions internationales, quelles que soient les variations dans leurs modalités de création $^{1}$, ont pendant longtemps constitué les cadres privilégiés d'application du droit pénal international. Par contre, la tendance actuelle met en avant le rôle primordial des instances étatiques dans la répression des crimes relevant du droit international.

Dans la littérature comme au sein du mouvement international des droits de l'homme, l'idée que «le droit pénal international trouverait son avenir au niveau national $»^{2}$ fait son chemin. La campagne en vue d'une adoption généralisée des règles de compétence universelle dans les législations nationales constitue l'une des manifestations de cette nouvelle orientation dans les discours et les pratiques sur la mise en œuvre du droit pénal international ${ }^{3}$. Cette nouvelle orientation justifie également, dans une certaine mesure, la préférence actuelle des tribunaux mixtes ou hybrides sur les tribunaux purement internationaux lorsque les Nations unies interviennent dans les processus de justice post-conflit. Les premiers offrent un potentiel plus élevé de renforcement des capacités des institutions locales, ultimes sphères de répression des crimes internationaux. Enfin, l'idée que la justice pénale internationale répond généralement mieux aux besoins des populations affectées par les crimes sous le Statut de Rome si elle est administrée localement justifie en partie le régime de complémentarité entre les instances nationales des États parties au Statut de Rome et la Cour pénale internationale (CPI) dans la répression des crimes internationaux. En effet, les règles de complémentarité sous l'empire du Statut de Rome consacrent la primauté des poursuites nationales, la CPI n'intervenant, en principe, que dans l'hypothèse d'un «manque de volonté et/ou incapacité » de l'État compétent à poursuivre 4 .

William W Burke-White emprunte la métaphore d'une «communauté de juridictions » [notre traduction] à Laurence R Helfer et Anne-Marie Slaughter pour ainsi décrire la situation dans laquelle une pluralité de juridictions ayant leurs sièges dans des espaces géographiques différentes ${ }^{5}$ sont simultanément impliquées dans

1 Le Tribunal militaire de Nuremberg a été établi sur base d'un traité conclu entre un groupe d'États, les alliés vainqueurs de la Deuxième Guerre mondiale. Voir Accord concernant la poursuite et le châtiment des grands criminels de guerre des Puissances européennes de l'Axe, 8 août 1945, 82 RTNU 281 (entrée en vigueur : 8 août 1945). Les tribunaux pénaux internationaux respectivement pour l'ex-Yougoslavie et pour le Rwanda ont été créés sur base de résolutions du Conseil de sécurité de l'ONU, agissant dans le cadre du Chapitre VII de la Charte des Nations unies. Voir Statut pour le Tribunal pénal international pour l'ex-Yougoslavie, 1993, Rés CS 827, Doc off CS NU, Doc NU S/RES/827; Statut du Tribunal pénal international pour le Rwanda, Rés CS 955, Doc off CS NU, Doc NU S/RES/955 (1994) [Résolution 955]. La Cour pénale internationale a été créée par traité multilatéral. Voir Statut de Rome de la Cour pénale internationale, 17 juillet 1998, A/CONF.183/9, 2187 RTNU 3854 (entré en vigueur : $1^{\text {er }}$ juillet 2002) [Statut de Rome].

2 L'expression vient d'Anne-Marie Slaughter et William Burke-White, « The Future of International Law is Domestic (or, The European Way of Law) » (2006) 47:2 Harv Int'1 LJ 327.

3 Amnistie International est particulièrement impliquée dans cette campagne. Amnesty International, " Universal Jurisdiction: A Preliminary Survey of Legislation Around The World - 2012 Update », en ligne : Amnesty International <www.amnesty.org $>$.

$4 \quad$ Statut de Rome, supra note 1, art 17(1)a).

5 C'est-à-dire nationales ou internationales. 
l'administration de la justice pénale internationale ${ }^{6}$. Davantage que la logique d'une « communauté de cours », le concept de « régime complexe » et les dynamiques qu'il implique offrent un cadre conceptuel d'analyse plus approprié des développements actuels vers une régionalisation de la justice pénale internationale en Afrique, sur fond de tensions entre l'Union africaine (UA) et certains États africains d'une part, la CPI et des États occidentaux d'autre part ${ }^{7}$. Matiangai Sirleaf est la première, d'après l'auteure, à appliquer le concept de " régime complexe » dans le domaine du droit pénal international, spécifiquement dans ce contexte ${ }^{8}$.

Avant les projets en cours sur le continent africain, les niveaux régional (continental) et sous-régional (zones géographiques généralement couvertes par les organisations couramment connues sous le nom de Communautés Économiques Régionales (CERs) étaient largement ignorés comme sphères d'application du droit pénal international. Pourtant, des observateurs avaient souligné, et cela sans anticipation de la crise actuelle que traverse la CPI, le potentiel unique d'une exploration régionaliste du droit pénal international dans la réalisation des objectifs majeurs de la justice pénale internationale, difficilement réalisables à une sphère plus globale d'administration de la justice pénale internationale?

La crise de légitimité sans précédent de la CPI sur le continent africain, culminant avec le retrait, en octobre 2016, de trois États du Statut de Rome, pourrait probablement tourner le regard des acteurs intéressés par la lutte contre l'impunité en Afrique vers le rôle potentiel des mécanismes régionaux dans la recherche d'une solution à ce problème ${ }^{10}$. Cet article épouse, mais va au-delà des opinions considérant ces juridictions comme des cadres alternatifs d'application du droit pénal international qui permettraient éventuellement de combler un vide dans le domaine de la lutte contre l'impunité des crimes les plus graves sur le continent africain, entrainé par la

6 William W Burke-White, « A Community of Courts: Toward a System of International Criminal Justice » (2002) 24:1 Mich J Int'1 L 1 (« [t]he emerging system of international criminal justice can be conceived as a community of courts, [...] a set of adjudicatory bodies in interdependent, selforganizing relationships. This emergent community of courts is engaged in a common endeavorensuring accountability for serious international crimes » à la p 3). Pour une exploration de la notion de communauté dans l'application du droit international en général, voir Laurence R Helfer et AnneMarie Slaughter, «Toward a Theory of Effective Supranational Adjudication » (1997) 107:2 Yale LJ 273; Anne-Marie Slaughter, « The Real New World Order » (1997) 76:5 Foreign Affairs 183.

7 Kal Raustiala et David G Victor, «The Regime Complex for Plant Genetic Resources » (2004) 58:2 Intl Organisation 277 («[...] an array of partially overlapping and nonhierarchical institutions governing a particular issue-area. Regime complexes are marked by the existence of several legal agreements that are created and maintained in distinct for a with participation of different sets of actors » à la p 279); Peter K Yu, « International Enclosure, the Regime Complex, and Intellectual Property Schizophrenia » (2007) 1:1 Mich State L Rev 1 («the salient, yet disturbing, characteristics of a regime complex are incoherence, inconsistency, and fragmentation " à la p 16; «strategic inconsistencies occur [...] when actors deliberately seek to create inconsistency via a new rule crafted in another forum in an effort to alter or put pressure on an earlier rule » à la p 17).

8 Matiangai VS Sirleaf, «Regionalism, Regime Complexes and the Crisis in International Criminal Justice » (2016) 54:3 Colum J Transnat'l L 699 à la p 705 [Sirleaf].

9 Voir principalement William W Burke-White, «Regionalization of International Criminal Law Enforcement: A Preliminary Exploration » (2003) 38:4 Tex Int'l LJ 729 [Burke-White, « Regionalization of International Criminal Law Enforcement »].

10 Voir en ce sens Stanley Ibe, «Regional Pathways for Preserving Justice in Africa » (23 novembre 2016), en ligne : IPI Global Observatory <www.theglobalobservatory.org>. 
crise. Une option régionaliste de la justice pénale internationale apporterait toujours, même dans les circonstances normales, un complément utile à la justice globale.

L'article est divisé en trois parties. La première consiste en une brève introduction sur le projet de justice transitionnelle au Burundi qui fournit le cadre d'application du droit pénal international pour le pays. Elle situe également la politique burundaise en matière de justice transitionnelle et de justice pénale internationale dans le cadre des débats et des pratiques internationaux dans ces domaines. La deuxième partie de l'article explore les avantages d'une approche régionaliste sur la justice pénale internationale. Enfin, la troisième partie de l'article propose une forme de coopération adéquate en matière d'intervention pénale internationale et, par la même occasion, une solution pour régler certains conflits de compétences éventuels entre des juridictions supranationales. Dans la logique d'une complémentarité positive entre les juridictions supranationales et à défaut de poursuites nationales, l'article propose que la juridiction supranationale ayant la plus grande "proximité géographique » avec le territoire du pays et des communautés affectés devrait bénéficier d'une primauté de compétence pour poursuivre les auteurs des crimes en question.

\section{Aperçu sur le projet et la politique de justice transitionnelle au Burundi}

Depuis bientôt deux décennies, le Burundi a enclenché un processus de justice transitionnelle pour tenter de mettre un terme à un demi-siècle d'impunité des crimes de masse qui ont marqué l'histoire du pays. Si le projet de justice transitionnelle au Burundi trouve ses fondements dans le contexte et l'histoire du pays, la politique de justice transitionnelle au Burundi s'inscrit également dans le contexte de l'évolution des idées et des pratiques internationaux sur la justice transitionnelle et la justice pénale internationale.

Le rapport de 2004 du Secrétaire général de l'ONU définit la justice transitionnelle comme

l'éventail complet des divers processus et mécanismes mis en œuvre par une société pour tenter de faire face à des exactions massives commises dans le passé, en vue d'établir les responsabilités, de rendre la justice et de permettre la réconciliation. ${ }^{11}$

Quoique la gamme des objectifs assignés à la justice transitionnelle varie largement dans la littérature, la découverte de la vérité ainsi que la détermination des responsabilités pénales comptent parmi les thèmes récurrents ${ }^{12}$. Les tribunaux et les

11 Rapport du Secrétaire général au Conseil de sécurité sur le rétablissement de l'état de droit et administration de la justice pendant la période de transition dans les sociétés en proie à un conflit ou sortant d'un conflit, Doc off CS NU, 2004, Doc NU S/2004/616 à la p 8.

12 Pour une revue de littérature, voir par ex Geneviève Parent, « Justice transitionnelle et maintien de la paix» (13 janvier 2012), en ligne: Réseau de recherche sur les opérations de paix $<$ www.operationspaix.net/134-resources/details-lexique/justice-transitionnelle-et-maintien-de-lapaix.html>. 
commissions de vérité et/ou réconciliation sont les principaux outils couramment déployés pour atteindre ces objectifs ${ }^{13}$. Étant donné l'objet de cet article, l'auteur se focalisera essentiellement sur les initiatives visant l'établissement des responsabilités pénales dans le contexte du Burundi.

\section{A. Fondements et cadre d'application du droit pénal international au Burundi}

\section{LE CONTEXTE DES CRIMES DE MASSE AU BURUNDI}

Successivement colonisé par l'Allemagne et la Belgique, le Burundi a accédé à l'indépendance le $1^{\text {er }}$ juillet 1962. Alors que le pays acquiert son indépendance dans le cadre d'un régime de monarchie constitutionnelle, celui-ci est vite renversé à l'issue d'un coup d'État militaire mené par le capitaine Michel Micombero, lequel se proclame président de la première République burundaise et dirigera le pays de 1966 à 1976. Micombero est renversé par le colonel Jean-Baptiste Bagaza en 1976. Ce dernier est lui-même écarté du pouvoir par le major Pierre Buyoya une fois encore par un coup d'État militaire, en 1987. L'histoire du Burundi d'après l'indépendance est dominée par un conflit politico-ethnique, opposant ses deux principales composantes ethniques, à savoir la majorité hutue (environ $85 \%$ ) et la minorité tutsie (autour de $15 \%$ ). En effet, chacun des régimes militaires successifs connait au moins une période de violences de masse liée à ces guerres fratricides, exception faite de la deuxième République (1976-1987). Les périodes de violences majeures se situent dans les années 1965, 1972, 1987, pour culminer avec la guerre civile qu'a connu le pays à partir des années 1994 .

L'ampleur et la fréquence de la violence au Burundi contrastent avec le peu d'intérêt tant de la part des décideurs internes que des acteurs internationaux dans le traitement des crimes du passé au Burundi. Écrivant au milieu de la guerre civile (1994 et suivants) et peu avant le génocide contre les Tutsis au Rwanda, René Lemarchand affirmait que «nulle part ailleurs en Afrique autant de violences n'ont tué autant de personnes à de si nombreuses occasions en un si petit espace qu'au Burundi d'après l'indépendance $»^{14}$ [notre traduction]. L'ONU est communément critiquée pour son inaction historique face aux violations graves, à grande échelle et répétitives des droits de l'homme dans le cadre du conflit burundais ${ }^{15}$. Cela dit, l'ONU a mandaté une série de missions d'investigation sur les crimes commis dans le contexte de certaines des périodes majeures de violence au Burundi. Même si ces différentes commissions n'étaient pas investies de compétences leur permettant de qualifier les crimes commis, au moins deux des rapports de mission évoquent

13 Ibid.

14 René Lemarchand, Burundi: Ethnic Conflict and Genocide, $2^{\mathrm{e}}$ éd, Cambridge, Cambridge University Press, 1996 à la p xxv.

15 Une des critiques émane d'un rapport rédigé au terme d'une des missions mandatées par les Nations unies mêmes, en l'occurrence le rapport dit Kalomoh, du nom de son auteur. Voir Conseil de Sécurité, Lettre datée du 11 mars 2005, adressée au Président du Conseil de sécurité par le Secrétaire général, Doc NU S/2005/158 (11 mars 2005) au para 19(d) [Rapport Kalomoh]. 
néanmoins la commission de crimes contre l'humanité, de crimes de guerre voire de génocide qui auraient été perpétrés dans le cadre du conflit ${ }^{16}$.

L'Accord d'Arusha pour la paix et la réconciliation au Burundi signé le 28 août 2000 est à l'origine du cadre légal et institutionnel de répression des crimes internationaux au Burundi ${ }^{17}$. Parmi les initiatives relatives à la poursuite des crimes les plus graves du passé, l'Accord d'Arusha prévoyait la mise en place d'un tribunal pénal international pour le Burundi chargé de poursuivre et réprimer les crimes de génocide, crimes de guerre et crimes contre l'humanité, commis dans le cadre du conflit burundais ${ }^{18}$. Toutefois, l'opportunité ainsi que les modalités de mise en place de cette instance restaient à déterminer par les autorités nationales, en collaboration avec l'ONU ${ }^{19}$. L'Accord d'Arusha recommandait également la «promulgation d'une législation contre le génocide, les crimes de guerre et autres crimes contre l'humanité et toute violation des droits de l'homme $»^{20}$. Une loi portant répression de ces crimes a été adoptée en mai 2003 pour donner suite à cette recommandation ${ }^{21}$. Dans la même optique, les juridictions nationales ont été dotées de compétences en matière de crimes internationaux à l'issue de la révision du Code pénal burundais, en avril $2009^{22}$. Des dispositions relatives à la compétence universelle pour les crimes internationaux ${ }^{23}$ ont été intégrées dans le Code pénal qui reprend, pour l'essentiel, les dispositions corrélatives du Statut de Rome, instrument que le Burundi avait d'ailleurs ratifié le 21 septembre $2004^{24}$.

16 Voir ONU, Sub-Commission on Prevention of Discrimination and Protection of Minorities, Revised and updated report on the question of the prevention and punishment of the crime of genocide, Doc off CES NU, 38 ${ }^{\mathrm{e}}$ sess, Doc CES E/CN.4/Sub.2/1985/6 (29 aout 1985) aux para 24, 36; Conseil de Sécurité, Lettre datée du 25 juillet 1996, adressée au Président du Conseil de Sécurité par le Secrétaire général, Doc NU S/1996/682 (1996) aux para 483, 496. Le premier traite des crimes commis durant la crise de 1972 tandis que le second est relatif aux massacres perpétrés pendant la guerre civile de 1993 et suivants.

17 Accord d'Arusha pour la paix et la réconciliation au Burundi, 28 aout 2000 (entrée en vigueur : 28 aout 2000) [Accord d'Arusha].

18 Ibid, protocole 1, art 6(11).

19 Ibid, art 6(10).

20 Ibid, art 6(9).

21 Loi $n^{\circ} 1 / 004$ du 8 mai 2003 portant répression du crime de génocide des crimes contre l'humanité et des crimes de guerre, 8 mai 2003.

22 Loi $n^{\circ} 1 / 05 d u 22$ avril 2009 portant révision du Code pénal, 22 avril 2009, art 171 et s. Les incriminations des crimes internationaux sont définies aux articles 195 à 203 du Code pénal burundais qui reprennent les dispositions corrélatives du Statut de Rome.

23 Ibid, art 8(2), 10(3).

24 Secrétaire général de l'ONU, Statut de Rome de la Cour pénale internationale, Rome, 17 juillet 1998 Burundi : ratification, C.N.936.2004.TREATIES-26, 21 septembre 2004. 


\section{B. La politique de justice transitionnelle au Burundi dans le contexte des pratiques et des débats internationaux sur la justice pénale internationale}

\section{LE MÉCANISME D’APPLICATION DU DROIT PÉNAL INTERNATIONAL PROPOSÉ POUR LE BURUNDI}

Jusqu'à récemment, le mécanisme judiciaire qui était proposé comme organe principal d'application du droit pénal international pour le Burundi, en l'occurrence un Tribunal Spécial mixte, pouvait se situer dans le cadre de l'évolution de la politique et des pratiques de l'ONU, lorsque l'organisation apporte son assistance aux pays en situation post-conflit. Le projet burundais s'inscrit dans le contexte où, d'une part, l'ONU a adopté une position de principe qui veut qu'elle n'endosse plus des accords politiques qui n'intégreraient pas des dispositions sur les mesures relatives à la lutte contre l'impunité des crimes de droit international ${ }^{25}$ et, d'autre part, la préférence en faveur des juridictions dites hybrides ou mixtes par rapport aux tribunaux purement internationaux comme mécanisme institutionnel de répression des crimes internationaux.

L'Accord d'Arusha s'écartait quelque peu de cette ligne d'idées puisqu'il envisageait plutôt la création d'un tribunal pénal international pour le Burundi. L'Accord d'Arusha aménageait toutefois une intervention ultérieure de l'ONU dans l'élaboration de la politique de justice transitionnelle au Burundi. Il conditionnait en effet la mise en place du tribunal envisagé aux conclusions d'un rapport par une commission internationale d'enquête qui serait mandatée par le Conseil de sécurité de l'ONU et établirait l'existence d'actes de génocide, de crimes de guerre et autres crimes contre l'humanité ${ }^{26}$. L'intervention de l'ONU dans ce processus a été effectuée en deux phases principales. Tout d'abord à travers les recommandations d'un rapport émanant de la mission ainsi envisagée en mai 2004; ensuite, à l'occasion des négociations ultérieures entre le gouvernement du Burundi et l'ONU sur les mécanismes de justice transitionnelle et les modalités de leur mise en place, tenues entre février 2006 et mars $2007^{27}$.

Conduite par l'ancien assistant du Secrétaire général de l'ONU aux affaires politiques, Tuliameni Kalomoh, la mission d'évaluation de l'ONU a séjourné au Burundi du 16 au 24 mai 2004. Au terme de sa mission, la délégation a proposé à la fois la mise en place d'un mécanisme de réconciliation ainsi que la création d'un mécanisme d'établissement des responsabilités pénales qui, aux yeux de ses membres, étaient plus appropriés dans le contexte du Burundi. S'agissant précisément du mécanisme judiciaire, le Rapport Kalomoh proposait la création d'une chambre criminelle intégrée au sein de l'appareil judiciaire burundais, spécialement

25 Priscilla Hayner, Negotiating Justice: Guidance for Mediators, Genève, Centre for Humanitarian Dialogue and the International Center for Transitional Justice, 2009 aux pp 8-9.

26 Accord d'Arusha, supra note 17.

27 Les différents documents en rapport avec ces négociations sont disponibles en ligne « Droit, pouvoir et paix au Burundi », en ligne : University of Antwerp <https:/www.uantwerpen.be/en/rg/institute-ofdevelop/centre-great-lakes/dpp-burundi/> [Droit, pouvoir et paix]. 
compétente pour traiter des crimes internationaux commis dans le passé du Burundi ${ }^{28}$. Selon les termes du rapport, la délégation devait tenir ainsi compte « de l'expérience acquise au cours de la dernière décennie pendant laquelle les Nations unies se sont consacrées à promouvoir la justice et l'État de droit ${ }^{29}$.

Par ailleurs, le Rapport Kalomoh revenait sur les critiques fréquemment émises à l'endroit des tribunaux ad hoc du modèle de celui pour l'ex-Yougoslavie et pour le Rwanda pour justifier le rejet de cette formule dans le cas du Burundi. En fin de compte, la commission affirmait s'inspirer de l'organisation et des modes de fonctionnement de la Chambre des crimes de guerre de la Cour de BosnieHerzégovine, alors en cours de création. Quoiqu'intéressant à bien des égards de l'avis même de la délégation, le modèle de la Sierra Leone, un tribunal spécial mixte siégeant sur le territoire du pays, a été écarté au motif qu'il n'était pas intégré au sein de l'appareil judiciaire sierra léonin. Ceci, selon les auteurs du rapport, pourrait limiter le potentiel du tribunal spécial au renforcement des capacités locales ${ }^{30}$. C'est pourtant le modèle de la Sierra Leone qui prévaudra comme principale source d'inspiration pour le Burundi au terme des négociations ultérieures menées entre le gouvernement burundais et l'ONU. Un organe judiciaire hybride était ainsi proposé sous l'appellation de Tribunal spécial ${ }^{31}$. La raison principale du changement de position est liée au fait que la société civile locale était particulièrement opposée à l'idée d'intégrer un mécanisme judiciaire spécial proposé au sein de l'appareil judiciaire burundais en proie à une profonde crise de confiance ${ }^{32}$.

\section{2. ÉTAT DES LIEUX DE LA POLITIQUE DE LUTTE CONTRE L'IMPUNITÉ DES CRIMES DE DROIT INTERNATIONAL AU BURUNDI}

Bien que la lutte contre l'impunité au Burundi soit rarement un sujet de débat dans la presse internationale, la récente décision prise en octobre 2016, par le gouvernement du Burundi, de se retirer du Statut de Rome a dominé l'actualité. Et pour cause. Aussi étonnante fut l'annonce surprise du processus de retrait du Statut de Rome que le rythme marathonien de la procédure. L'Assemblée nationale du Burundi a adopté, le 12 octobre 2016, à une majorité écrasante (94/110), un projet de loi portant retrait du Statut de Rome. Le texte de loi $^{33}$ a été promulgué par le président de la République le 18 octobre 2016, soit moins d'une semaine après son adoption et cela alors même que la Constitution de la République du Burundi accorde au président un délai de 30 jours à compter de la date de réception d'une loi adoptée par le parlement

\footnotetext{
Rapport Kalomoh, supra note 15 au para 52.

Ibid au para 57.

Ibid aux para 59-60.

Droit, pouvoir et paix, supra note 27.

32 Forum pour le Renforcement de la Société civile (FORSC), Mémorandum présenté par la société civile sur les mécanismes proposés par la commission d'évaluation des Nations unies sur les crimes commis au Burundi, Bujumbura, Juin, 2005.

33 Loi $n^{\circ} 1 / 14 d u 18$ octobre 2016 portant retrait de la République du Burundi du Statut de Rome, 18 octobre 2016.
} 
aux fins de sa promulgation ${ }^{34}$. Le fait est que l'initiative du projet émanait en réalité du bureau de la Présidence même ${ }^{35}$.

Officiellement, les autorités burundaises ont tenté de justifier la décision en la liant au mouvement de contestation contre la politique d'intervention de la CPI en Afrique fréquemment dénoncée par les dirigeants africains comme étant «biaisée $»^{36}$. Deux autres pays africains, en l'occurrence l'Afrique du Sud et la Gambie ont, il est vrai, pris des décisions similaires quelques jours plus $\operatorname{tard}^{37}$. Toutefois, le cas du Burundi se distingue en raison du contexte de la crise politique et humanitaire ainsi qu'au regard de la situation déplorable des droits de l'homme dans le pays. La démarche est intervenue, semble-t-il, en réaction à une série de rapports de sources diverses, y compris de l'ONU, accusant certains parmi les plus hautes autorités burundaises de violations graves des droits de l'homme dans le cadre de la gestion de la crise que traverse le pays depuis la réélection du président Pierre Nkurunziza à un troisième mandat contesté en juillet 2015. Un de ces rapports, publié à deux semaines de la procédure de retrait et rédigé par un groupe de trois experts indépendants mandatés par les Nations unies, fait état de probables crimes contre l'humanité et d'« un grand danger de génocide [...] étant donné l'histoire du pays » ${ }^{38}$. Le rapport attribue la responsabilité « de la majeure partie des violations » à des agents officiels ou à des personnes dont la responsabilité des actes peut être attribuée au gouvernement ${ }^{39}$. Déjà en avril 2016, la procureure de la CPI, Fatou Bensouda, avait annoncé l'ouverture d'un examen préliminaire sur la situation au Burundi à la suite «de communications et de rapports faisant état de meurtres, d'emprisonnements, d'actes de torture, de viols et autres formes de violence sexuelle, ainsi que de cas de disparitions forcées $»^{40}$. Il n'est donc pas étonnant que le communiqué du gouvernement à la base du projet prenne violemment à partie les auteurs du rapport ainsi que la procureure elle-même ${ }^{41}$. Au reste, comme le professeur Pacifique

34 Loi $n^{\circ} 1 / 010$ du 18 mars 2005 portant promulgation de la Constitution de la République du Burundi, 18 mars 2005, art 197.

35 Gouvernement du Burundi, communiqué, «Le Conseil des Ministres s'est réuni ce jeudi 6 Octobre 2016 à Gitega sous la présidence de son Excellence le Président de la République, Monsieur Pierre Nkurunziza » (6 octobre 2016), en ligne : Gouvernement du Burundi <www.burundi.gov.bi/ spip.php?article1534> [Conseil des Ministres].

36 Ibid.

37 Le 19 octobre 2016 dans le cas de l'Afrique du Sud et le 25 octobre dans le cas de la Gambie. Sewell Chan et Marlise Simons, «South Africa to Withdraw from International Criminal Court », The New York Times (21 octobre 2016), en ligne: The New York Times <www.nytimes.com>; « Gambia announces withdrawal from International Criminal Court», Reuters (25 octobre 2016), en ligne : Reuters $<$ www.reuters.com $>$. Au lendemain de la défaite électorale de Yajay Jameh suivie de son exil, le retour de la Gambie parmi les États parties à la CPI a été l'une premières mesures annoncées par les nouvelles autorités. Voir Elise Keppler, « Gambia rejoins ICC: South Africa, Burundi now outliers on Exit » (17 février 2017), en ligne : Human Rights Watch <www.hrw.org>.

38 Conseil des droits de l'homme, Rapport de l'enquête indépendante des Nations unies sur le Burundi (EINUB) établie conformément à la résolution $S-24 / 1$ du Conseil des droits de l'homme, Doc off AG NU, $33^{\mathrm{e}}$ sess, Doc NU AHRC/33/37 (2016), aux para 123-124.

39 Ibid au para 125.

40 Cour pénale internationale, communiqué, « Déclaration du Procureur de la Cour pénale internationale, Mme Fatou Bensouda, à propos de l'examen préliminaire entamé dans le cadre de la situation au Burundi » (25 avril 2016), en ligne : CPI <www.icc-cpi.int $>$.

41 Conseil des ministres, supra note 35. 
Manirikaza le fait remarquer, par ailleurs, le Burundi ne comptait même pas auparavant parmi les États africains qui dénonçaient régulièrement la politique d'intervention de la CPI en Afrique ${ }^{42}$.

La décision des autorités burundaises s'interprète encore davantage à la lumière des prises de positions officielles en matière de justice transitionnelle au Burundi, s'agissant spécifiquement des mesures relatives à la lutte contre l'impunité ${ }^{43}$. En effet, au fur et à mesure de la progression du projet vers la mise en place effective des mécanismes de justice transitionnelle, les autorités officielles n'ont cessé d'exprimer leur hostilité à l'égard d'un traitement judiciaire des dossiers liés aux crimes du passé. À plusieurs occasions durant les négociations avec l'ONU et avant que le partenariat sur le projet ne soit en réalité rompu, le gouvernement du Burundi marquait sa préférence exclusive en faveur d'approches non judiciaires comme les procédures centrées sur l'octroi d'amnisties ou du pardon, inspirées explicitement des travaux de la Commission vérité et réconciliation en Afrique du $\mathrm{Sud}^{44}$. En mai 2014, une loi création d'une Commission vérité et réconciliation (CVR) a été promulguée sans mention d'aucun mécanisme judiciaire dans le texte ${ }^{45}$. Les groupes de défense de droits de l'Homme ont interprété cette omission comme l'expression de la volonté politique d'écarter définitivement la mise en place du Tribunal spécial initialement envisagé comme mécanisme principal de répression des crimes les plus graves commis dans le cadre du conflit burundais. Les activités de la CVR ont officiellement été lancées le 4 mars 2016, mais il serait simplement naïf de s'imaginer que la CVR ait effectivement été en mesure de réaliser son mandat dans le contexte sociopolitique du pays. En définitive, les perspectives internes d'une justice transitionnelle effective, quel que soit au reste l'aspect du projet (judiciaire ou non judiciaire), sont difficiles à envisager dans un proche avenir.

Il ne fait pas de doute, par ailleurs, que les instances judiciaires locales accuseront toujours un déficit de compétences techniques pour traiter des dossiers impliquant des crimes de droit pénal international, à supposer même l'existence d'un contexte politique idéalement favorable. Le Rapport Kalomoh mentionnait déjà le déficit de qualification et de formation des praticiens de la justice comme une des « défectuosités endémiques » du secteur judiciaire ${ }^{46}$. La remarque est particulièrement pertinente lorsqu'il s'agit, pour les juges burundais, d'appliquer le droit pénal international dans la mesure où le législateur burundais a choisi de réprimer les crimes internationaux effectivement sous leur qualification en tant que crimes internationaux

42 Pacifique Manirakiza, « Le retrait du Burundi de la Cour pénale internationale et ses implications », en ligne : Association de réflexion et d'information sur le Burundi $<$ www.arib.info $>$.

43 Voir dans le même sens Astrid Jamar, «Transitional Justice Battlegrounds: Another Bad Week in Burundi » (22 octobre 2016), en ligne : Justice in Conflict <www.justiceinconflict.org>.

44 Il s'agissait en réalité des positions du parti au pouvoir le CNDD-FDD, systématiquement entérinées officiellement. Voir par ex CNDD-FDD, « Mémorandum du parti CNDD-FDD sur la Commission vérité et réconciliation et le Tribunal spécial» (5 mai 2007). Pour une analyse détaillée, voir Stef Vandeginste, «Bypassing the Prohibition of Amnesty for Human Rights Crimes Under International Law: Lessons Learned from the Burundi Peace Process » (2011) 29:2 Nethl QHR 189.

45 Loi $n^{\circ} 1 / 18$ du 15 mai 2014 portant création, mandat, composition, organisation et fonctionnement de la commission vérité et réconciliation, 15 avril 2006.

46 Rapport Kalomoh, supra note 15 au para 50. 
et non comme des crimes de droit commun ${ }^{47}$.

\section{Les avantages d'une approche régionaliste en matière de justice pénale internationale}

L'administration de la justice pénale internationale par une juridiction physiquement proche des lieux de commission des crimes peut mieux contribuer à la réalisation d'un certain nombre d'objectifs d'une importance cruciale dans les contextes post-conflit. La reconstitution des liens sociaux brisés par le conflit, à travers une justice restauratrice ainsi que le renforcement des capacités des institutions locales dans la prévention et la lutte contre l'impunité sont les avantages communément relevés. L'application du droit pénal international dans un cadre régional peut offrir un avantage également important, lié à l'opportunité unique de réprimer des crimes d'une préoccupation particulière pour les populations de la région.

\section{A. Un potentiel plus élevé pour la reconstruction de l'État de droit et la réconciliation dans les sociétés divisées}

1. CONTRIBUTION À UNE JUSTICE RÉCONCILIATRICE

Les contextes post-conflits posent des défis particuliers à la justice pénale internationale. Un de ces défis tient à la nécessité d'intégrer des objectifs propres à ces contextes, mais inhabituels parmi les buts de la justice pénale classique de tradition libérale occidentale. En plus de sa fonction rétributive initiale, elle doit aménager des mesures visant entre autres la réconciliation. Ce tempérament est imposé par les réalités de terrain dans les sociétés divisées par des conflits intercommunautaires. Dans la plupart des cas, les auteurs des crimes et les victimes sont condamnés à vivre en permanence ensemble dans des relations de voisinage et d'interdépendance inévitables. C'est sans doute dans le contexte d'une telle réalité au Rwanda qu'il faut interpréter, en partie, cet aspect inhabituel dans les textes

47 L'application du droit pénal international constitue toujours un défi pour les juges nationaux, même les mieux formés. Voir à ce sujet Kevin Jon Heller, The Nuremberg Military Tribunals and the Origins of International Criminal Law, Oxford, Oxford University Press, 2011 aux pp 242-249. Un des défis à cet égard est lié au fait que, contrairement aux infractions de droit commun avec lesquels les juges nationaux sont familiers, la qualification des crimes internationaux exige, en plus de l'établissement des éléments moral et matériel de l'infraction, la détermination d'un cadre contextuel des crimes. Kevin Jon Heller donne à titre d'illustration, le fait qu'une haute juridiction comme la Cour suprême du Canada a pris des décennies pour établir une jurisprudence conforme au droit pénal international. Il souligne combien les défis seraient encore plus grands pour les juges des pays du Sud si les lois qu'ils appliquent réprimaient les faits constitutifs de crimes internationaux sous cette qualification au lieu de les incriminer comme des crimes de droit commun. Voir Kevin Jon Heller, «A Sentence-Based Theory to Complementarity » (2012) 53:1 Harv Intl LJ 201 [Heller, « Theory to Complementarity »]. Voir aussi Sharon A Williams et William A Schabas, "Article 17: Issues of Admissibility » dans Otto Triffterer, dir, Commentary on the Rome Statute of the International Criminal Court: Observers' Notes, Article by Article, $2^{\mathrm{e}}$ ed, Oxford, Hart, 2008 à la p 605. 
fondateurs des tribunaux internationaux, mais figurant dans la Résolution 955 instituant le Tribunal pénal international pour le Rwanda (TPIR). Selon les propos du Conseil de sécurité de l'ONU, « dans les circonstances particulières qui [régnaient] au Rwanda ", les poursuites pénales permettraient non seulement de mettre fin aux crimes internationaux, «mais contribueraient également au processus de réconciliation nationale $»^{48}$.

En réalité, la réconciliation est un objectif difficilement à la portée d'une juridiction de la nature du TPIR. Une opinion au sein de la littérature académique principalement d'obédience occidentale soutient, il est vrai, la thèse des vertus réconciliatrices du procès pénal, de par même l'établissement des responsabilités individuelles par le jugement. À ce propos, Antonio Cassese écrit ainsi :

Les procès pénaux établissent les responsabilités individuelles en remplacement d'une responsabilité collective, c'est-à-dire qu'ils établissent que non pas tous les Allemands étaient impliqués dans l'Holocauste, ou les Turcs dans le génocide arménien, ou tous les Serbes, musulmans, Croates, ou Hutus, mais les auteurs individuellement [...] le procès dissipe le désir de vengeance parce que lorsque la cour applique au bourreau la peine qu'il mérite, alors le cri des victimes au châtiment est entendu [...] les victimes sont prêtes à se réconcilier avec leurs anciens bourreaux, parce qu'elles savent que ces derniers ont payé leurs crimes; une vérité [documentation] totale fiable sur les atrocités est établie de telle sorte que les générations futures seront informées de ce qui s'est passé. ${ }^{49}$ [Notre traduction]

Pourtant, des études empiriques remettent sérieusement en cause cette $\operatorname{assertion}^{50}$. Une limite des juridictions internationales sous l'angle de la justice réconciliatrice communément relevée tient à leur éloignement des communautés affectées au moment où une justice réconciliatrice est largement tributaire de la place et de l'opportunité des communautés touchées de participer dans les procédures pénales. C'est pour souligner l'importance de cet aspect que des observateurs insistent sur l'avantage que procurait le rapprochement géographique du TPIR du territoire du Rwanda permettant à la procureure Louise Arbour de délocaliser des audiences d'Arusha (Tanzanie, siège du TPIR) à Kigali, la capitale du Rwanda ${ }^{51}$. En contraste, le Tribunal pénal international pour l'ex-Yougoslavie (TPIY) est entre autres critiqué

48 Résolution 955, supra note 1 à la $\mathrm{p} 1$.

49 Antonio Cassesse, «Reflection on International Criminal Law » (1998) 61:1 Mod L Rev 1 à la p 6 (« [t]rials establish individual responsibility over collective assignation of guilt, ie, they establish that not all Germans were responsible for the Holocaust, not all Turks for the Armenian genocide, nor all Serbes, Muslims, Croats, or Hutus but individual perpetrators [...] justice dissipates the call for revenge, because when the Court metes out to the perpetrator his just deserts, then victims' calls for retribution are met [...] victims are prepared to be reconciled with their erstwhile tormentors, because they know that the latter have now paid for their crimes; a fully reliable record is established of atrocities so that the future generations can remember and be made fully cognisant of what happened " [italiques dans l'original]).

50 Dans le cas du Rwanda, voir par ex Mark Drumbl, «Sclerosis: Retributive Justice and the Rwandan Genocide » (2000) 2:3 Punishment and Society 287 [Drumbl].

51 Payam Akhavan, « Beyond Impunity: Can International Criminal Justice Prevent Future Atrocities? » (2001) 95:1 Am J Int'l L 7 à la p 25. 
du fait qu'il offrait difficilement cette option ${ }^{52}$.

Au-delà de ces défis d'ordre pratique, des considérations idéologiques limitent tout autant le potentiel de ce genre de tribunaux sous l'angle qui nous occupe. Du point de vue de la victime, les procès pénaux internationaux incarnent la critique de Hannah Arendt à l'égard du procès pénal qu'elle dépeint ainsi :

$[u] n$ procès [pénal] ressemble à une pièce en ce sens que les deux débutent et se terminent toujours avec l'auteur et non la victime [...] et s'il [le bourreau] doit souffrir, il doit souffrir pour ce qu'il a fait et non pour la souffrance qu'il a infligée aux autres. ${ }^{53}$ [Notre traduction.]

La critique reste valable à propos des procès plus récents dans l'histoire de la justice pénale internationale ${ }^{54}$.

Une juridiction régionale permettrait bien évidemment, de par sa situation géographique, de combler les lacunes de la justice pénale internationale résultant de l'éloignement géographique des juridictions internationales des lieux de commission des crimes. En Afrique spécifiquement, il y a, en outre, de fortes chances qu'un traité régional permette également de contourner les obstacles idéologiques à l'encontre d'une procédure pénale plus ouverte à l'implication communautaire. Comme Kai Ambos le rappelle, les sociétés africaines en général mettent en avant une justice pénale restauratrice orientée vers la réconciliation et le rétablissement des liens sociaux plutôt qu'une justice «stigmatisante» exclusivement centrée sur le criminel $^{55}$. Comme nous pourrons le voir plus loin, cette conception africaine de la justice pénale à laquelle Kai Ambos fait référence transparaît effectivement à travers les documents relatifs aux projets d'établissement de mécanismes régionaux de droit pénal international et de justice transitionnelle en Afrique ${ }^{56}$.

\section{CONTRIBUTION AU RENFORCEMENT DES CAPACITÉS LOCALES}

Le renforcement des capacités locales occupe incontestablement une place centrale dans la définition des politiques de justice pénale internationale destinées aux sociétés en situation post-conflit. Comme nous l'avons relevé dans la section précédente, il s'agit d'un facteur déterminant dans la conception des mécanismes de

52 Wiliam Burke-White déplore l'invisibilité du TPIY aux yeux de nombreuses victimes des violences sexuelles dans le cadre du procès Kunarac. Voir Le Procureur c Kunarac, IT-96-23-T \& IT-96-23/1-T, Jugement (22 février 2001) (Tribunal pénal international pour l'ex-Yougoslavie, Chambre de première instance), cité dans Burke-White, "Regionalization of International Criminal Law Enforcement », supra note 9 à la p 735.

53 Hannah Arendt, Eichmann in Jerusalem: A Report on the Banality of Evil, New York, Viking Press, 1964 à la p 8.

54 Drumbl, supra note 50 à la p 294 commentant les procès pénaux au Rwanda et devant le TPIY, en particulier l'affaire Tadic.

55 Voir Kai Ambos « Expanding the Focus of the "African Criminal Court" » dans Yvonne McDermott, Niamh Hayes et William A Schabas, dir, The Ashgate Research Companion to International Criminal Law : Critical Perspectives, Burlington, Ashgate, 2013, 499 aux pp 527-528 [Ambos].

56 Infra note 151. 
droit pénal international appropriés dans de tels contextes. Certains vont même audelà des contextes des tribunaux spécifiquement mis en place pour traiter des situations post-conflit pour proposer que le renforcement des capacités locales des États soit une mission essentielle, lorsque c'est la CPI qui intervient.

Par exemple, William W Burke-White soutient que la CPI devrait envisager une politique d'intervention directe dans le renforcement des capacités locales des États où elle envisage des poursuites, y compris, le cas échéant, à travers la formation des praticiens locaux ${ }^{57}$. Cette approche traduirait, selon l'auteur, une politique de « complémentarité positive » entre la CPI et les États parties au Statut de Rome dans la répression des crimes relevant de la compétence de la CPI. L'idée d'une assistance directe de la CPI aux instances étatiques ne fait cependant pas l'unanimité. Contrairement à la proposition de William W Burke-White, Carey Shenkman suggère plutôt que la CPI se limite à un rôle de « catalyseur » éventuel des réformes nationales sans pour autant fournir une assistance directe aux États ${ }^{58}$. Dans une telle perspective, les réformes éventuelles résulteraient indirectement des poursuites pénales entreprises par la CPI, voire des menaces de poursuites dans les États en question. L'idée ici est que les pays visés entreprendraient des réformes dans le sens d'aligner leurs cadres légaux et institutionnels de répression des crimes prévus dans le Statut de Rome aux exigences internationales dans le dessein de préserver la primauté des poursuites pénales que l'article $17 \mathrm{du}$ Statut de Rome garantit aux États, mais moyennant des conditions $^{59}$. Il s'agirait précisément, de la part de ces États, de se prémunir contre les risques d'être qualifiés d'États "manquant de volonté et/ou incapables de mener à bien des poursuites pénales » au sens de l'article 17 du Statut de Rome et de perdre, par conséquent, dans le contexte des exceptions d'irrecevabilité des affaires devant la $\mathrm{CPI}^{60}$.

Quels que soient les mérites de la proposition, les limites des tribunaux internationaux sur le modèle de la CPI ou des tribunaux pénaux internationaux comme le TPIY ou le TPIR sous cet aspect sont communément admises parmi les acteurs de la justice pénale internationale. Dans le cas du Timor oriental, par exemple, c'est principalement par cet argument que le Secrétaire général de l'ONU justifiait l'abandon de la proposition d'un tribunal pénal international pour un mécanisme judiciaire plutôt mixte qui contribuerait mieux au renforcement des structures

57 William Burke-White, « Proactive Complementarity: The International Criminal Court and National Courts in the Rome System of International Justice» (2008) 49:1 Harv Int'l LJ 53 aux pp 85 et s [Burke-White, «Proactive Complementarity »]. Voir également Lisa J Laplante, « The Domestication of International Criminal Law: A Proposal for Expanding the International Criminal Court's Sphere of Influence » (2010) 43:3 John Marshall L Rev 635 à la p 636.

58 Carey Shenkman, «Catalyzing National Judicial Capacity: The ICC's First Crimes Against Humanity Outside Armed Conflict» (2012) 87:4 NYUL Rev 1210 [Shenkman].

59 Voir par ex Yvonne M Dutton, « Explaining State Commitment to The International Criminal Court: Strong Enforcement Mechanisms as a Credible Threat » (2011) 10:3 Wash U Global Stud L Rev 477, cité dans Shenkman, supra note 58 à la p 1233 .

60 C'est ainsi que les gouvernements kenyan et libyen, par exemple, avaient entrepris une série de réformes dans la perspective de démontrer la volonté et la capacité des autorités nationales de mener véritablement à bien des poursuites nationales et ainsi faire échec aux procédures devant la CPI. Pour plus de détails ainsi qu'une analyse critique, voir Shenkman, supra note 58 aux pp 1235-1239. 
judiciaires nationales avec l'assistance de l'ONU ${ }^{61}$. Le potentiel élevé des tribunaux mixtes ou hybrides dans le renforcement des capacités locales découle à la fois de leur présence physique localement ainsi que d'une combinaison d'éléments internationaux et locaux dans leurs modes d'organisation et de fonctionnement ${ }^{62}$.

Dans la même optique, une approche régionaliste sur la justice pénale internationale offre des opportunités comparables. William W Burke-White résume ainsi la dynamique de fonctionnement de celles-ci : «les juridictions régionales comprendraient des procureurs, des juges et du personnel en provenance [des pays] de la région, formant du coup ceux qui retourneront probablement travailler au sein des systèmes judiciaires nationaux $\rangle^{63}$. [Notre traduction]

La Cour de justice de l'EAC (EACJ) en est un exemple concret. L'EACJ est l'organe judiciaire de l'EAC et a son siège actuel à Arusha en Tanzanie ${ }^{64}$. Elle comprend une section de première instance et une section d'appel ${ }^{65}$. La section de première instance compte dix juges, tandis que celle d'appel en est composée de cinq $^{66}$. Les juges sont désignés par le Sommet des chefs d'États et de gouvernements des États membres de l'EAC sur base d'une liste de nominations préalablement soumises par les États ${ }^{67}$, qui disposent par ailleurs d'un droit d'égale représentativité au sein de chacune des deux chambres de l'EACJ ${ }^{68}$. L'impact de l'EACJ au niveau du renforcement des capacités des pays membres de l'EAC peut être instrumental. C'est le cas s'agissant, par exemple, d'un juge qui termine son mandat de juge et rentre travailler comme cadre au sein d'un service national de législation au Burundi ou encore d'un autre qui rentre pour enseigner dans une faculté de droit ${ }^{69}$. Il y a, par ailleurs, tout lieu de croire que l'impact de ce type de juridictions dans le renforcement des capacités locales pourrait être encore plus direct, dans la logique de ce que souhaitait William Burke White de la CPI. Rien n'empêcherait aux États qui négocient un traité régional de prévoir que les juges régionaux interviendraient dans la formation des juges et des praticiens locaux ${ }^{70}$. Les obstacles idéologiques à ce genre d'arrangements ne se poseraient pas nécessairement dans le cadre d'un traité régional africain.

61 Voir Suzannah Linton, «Cambodia, East Timor and Sierra Leone: Experiments in International Justice » (2001) 12:2 Crim LF 185 aux pp 202 et s.

62 Ibid.

63 Burke-White, « Regionalization of International Criminal Law Enforcement », supra note 9 à la p 735.

64 Traité instituant la Communauté de l'Afrique de l'Est, 30 novembre 1999, 2145 RTNU 255 (entrée en vigueur: 7 juillet 2000), art 9 [Traité de l'EAC]. La page internet de l'EACJ indique qu'Arusha constitue le siège temporaire de la Cour. La localité du siège de la Cour reste à déterminer par le Sommet des des Chefs d'États et de Gouvernement des États membres de la Communauté (art 47).

65 Ibid, art 23(2).

66 Ibid, art 24(2)

67 Ibid, art 24(1).

68 Ibid, art 24(1)(a)(b), 24(2).

69 Les anciens juges Jean-Bosco Butasi ainsi que le professeur Laurent Nzosaba se trouvent respectivement dans ces situations au moment où nous écrivons.

70 Voir aussi Burke-White, « Regionalization of International Criminal Law Enforcement », supra note 9 à la p 735 («[p]articularly if regional courts were given a specific mandate of engaging with and training national courts, they could offer a powerful tool for post-conflict reconstruction »). 


\section{B. Une opportunité unique de réprimer des crimes d'une préoccupation particulière pour la région}

Un traité négocié à l'échelle régionale peut offrir aux États impliqués, une occasion unique de réprimer des crimes d'une préoccupation particulière aux populations de la région. Une analyse comparée des compétences matérielles de la CPI en vertu du Statut de Rome, d'une part, et de la Section de droit pénal international (SDPI) telle qu'envisagée par le Protocole portant amendements au Protocole portant Statut de la Cour africaine de justice et des droits de l'homme ${ }^{71}$, d'autre part, permet d'illustrer ce propos. Dans la même logique, une analyse de l'évolution de la pratique de l'EACJ dans les domaines de la bonne gouvernance, de l'État de droit et des droits de l'homme permet de conclure qu'un organe de droit pénal international au sein de l'EAC suivrait la même trajectoire.

\section{ANALYSE COMPARÉE DES COMPÉTENCES DE LA SDPI ET DE LA CPI}

L'adoption du Protocole de Malabo a été entourée de vives polémiques et suscitée des controverses dans la littérature, liées aux circonstances du projet. En effet, le Protocole a été adopté dans le contexte d'une crise diplomatique entre l'UA d'une part, certains pays occidentaux et la CPI d'autre part, à la suite d'une série de poursuites contre des officiels africains, soit auprès des juridictions étatiques en Europe, soit devant la CPI. Des critiques ont alors été émises que le projet de création d'un organe de droit pénal international au sein des structures de l'UA équivaudrait à une tentative de court-circuiter toute intervention future de la CPI sur le continent ${ }^{72}$. On est même allé jusqu'à prétendre l'illégalité du projet ${ }^{73}$.

Pourtant, les débats sur le projet de création d'une juridiction de droit pénal international en Afrique remontent loin dans l'histoire de l'organisation panafricaine $^{74}$. L'idée avait par ailleurs été avancée dans la littérature sur la justice

71 Protocole portant amendements au Protocole portant Statut de la Cour africaine de justice et des droits de l'homme, $1^{\mathrm{er}}$ juillet 2008 [Protocole de Malabo].

72 Mutoy Mubiala, par exemple, estime que la juridiction envisagée fonctionnerait essentiellement comme un «écran » entre les instances nationales et régionales africaines d'une part et la CPI d'autre part. Mutoy Mubiala, «Chronique de droit pénal de l'Union Africaine. L'élargissement du mandat de la Cour africaine de Justice et des droits de l'homme aux affaires de droit international pénal » (2014) 85:3 Rev IDP 749 à la p 757 [Mubiala].

73 Chacha Bhoke Murungu, «Towards a Criminal Chamber in the African Court of Justice and Human Rights » (2011) 9:5 J Int'l Criminal Justice 1067. Voir cependant Ademola Abass, « Prosecuting International Crimes in Africa: Rationale, Prospects and Challenges » (2013) 24:3 Eur J Int'l L 933 aux pp 941-942 [Abass, « International Crimes »].

74 Ademola Abass fait remonter les premières discussions sur le projet dans les années 1970 dans le contexte de l'adoption de la Charte africaine des droits de l'homme et des peuples, 27 juin 1981, 1520 RTNU 217 (entrée en vigueur : 21 octobre 1986) [Charte africaine]. Une proposition de création d'une juridiction de droit pénal international figurait ainsi dans les travaux préparatoires de la Charte africaine, spécialement en rapport avec la répression du crime d'apartheid. Voir Abass, « International Crimes ", supra note 73 à la p 936, citant Keba M'Baye, Draft African Charter on Human and Peoples' Rights, OAU Doc CAB/LEG/67/1 au para 4. 
pénale internationale ${ }^{75}$. Le projet trouve sans nul doute une base légale dans l'Acte constitutif de l'Union africaine ${ }^{76}$, plus précisément dans ses articles 3(h), 4(h) et $4(\mathrm{o})^{77}$. L'article 4(h) autorise en effet l'organisation panafricaine à intervenir dans un État membre « dans certaines circonstances graves, à savoir : les crimes de guerre, le génocide et les crimes contre l'humanité $\rangle^{78}$.

Certes, les tensions entre l'UA et le monde occidental ont servi de catalyseur du projet ${ }^{79}$. Toutefois, limiter le projet au seul contexte de cette crise occulte une partie importante de son contexte historique. Les opinions dans ce courant avancent ainsi une analyse parcellaire du Protocole de Malabo. Pourtant, une analyse plus objective du texte permet de déceler une série d'innovations intéressantes, comparées au Statut de Rome et aux textes créateurs des juridictions de droit pénal international qui ont existé à ce jour ${ }^{80}$.

S'agissant précisément de la compétence matérielle de la SDPI qui nous intéresse ici, particulièrement dans une perspective comparative, la compétence de la CPI apparait «limitée aux crimes les plus graves » qui «touchent l'ensemble de la communauté internationale » pour reprendre les termes du Statut de Rome ${ }^{81}$. Les seuls crimes qui atteindraient ce seuil de gravité sont ceux qui constituent la trilogie traditionnelle des crimes internationaux, à savoir le crime de génocide, le crime contre l'humanité ainsi que les crimes de guerre auquel s'est ajouté le crime d'agression à l'issue de la conférence de Kampala en 2010. De son côté, l'article 28A du Protocole de Malabo reprend la liste des quatre crimes y compris leurs éléments constitutifs ${ }^{82}$, tout en y ajoutant dix incriminations, à savoir

75 Voir par ex Pacifique Manirakiza, « The Case for an African Criminal Court to Prosecute International Crimes Committed in Africa » dans Vincent Nmehielle, dir, Africa and the Future of international criminal justice, The Hague, Eleven, 2012, 375.

76 Acte constitutif de l'Union africaine, 11 juillet 2000, 2158 RTNU 3 (entrée en vigueur : 26 mai 2001) [Acte constitutif de l'UA].

77 Ibid.

78 C'est à l'occasion de la Conférence des chefs d'État et de gouvernement de l'Union africaine tenue à Banjul du 1 au 2 juillet 2006 que les chefs d'États et de gouvernement africains ont exploité, pour la première fois, les dispositions précitées sur la base d'un rapport d'experts juristes produit dans le cadre de la gestion du dossier Hissène Habré. Les dirigeants africains ont alors estimé que « les crimes reprochés à Hissène Habré (crimes contre l'humanité, crimes de guerre et actes de torture) sont pleinement de la compétence de l'Union africaine ». Ils se sont néanmoins heurtés à un obstacle pratique à savoir que l'UA ne disposait pas encore d'un organe judiciaire de droit pénal international. Par conséquent, ils ont décidé de confier le procès contre Hissène Habré à l'État sénégalais qui poursuivrait le suspect pour le compte de l'UA. Voir Conférence de l'Union africaine, Décision sur le Procès d'Hissène Habré et l'Union africaine, $7^{\mathrm{e}}$ sess, Doc Assembly/AU/Dec.127 (VII) (2006) au para 5(i), en ligne : <www.africa-union.org/Official documents/Assemblee\%20fr/ASS06b.pdf>.

79 Au sujet de la théorie des crises comme catalyseur dans l'évolution du droit international, voir par ex Benjamin Authers et Hilary Charlesworth, "The Crisis and the Quotidian in International Human Rights Law » (2013) 44 Nethl YB Int'1 L 19 à la p 25, cité dans Sirleaf, supra note 8 à la p 709. Benjamin Authers et Hilary Charlesworth se focalisent sur le droit international des droits de la personne et montrent comment l'idée de crise sert souvent de catalyseur d'action dans ce domaine.

80 Pour une analyse plus détaillée, voir par ex Sirleaf, supra note 8 à la p 755.

81 Statut de Rome, supra note 1, art 5.

82 Au sujet des éléments constitutifs des crimes de génocide, de crimes contre l'humanité et de crimes de guerre, comparer les articles 28B, 28C et 28D du Protocole de Malabo, supra note 71 d'une part et les articles 6 et suivants du Statut de Rome, supra note 1. Le crime d'agression reste à définir sous le régime du Statut de Rome. 
[...] le crime relatif au changement anticonstitutionnel de gouvernement, la piraterie, le terrorisme, le mercenariat, le crime de corruption, le blanchiment d'argent, la traite des personnes, le trafic illicite de stupéfiants, le trafic illicite de déchets dangereux ainsi que l'exploitation illicite des ressources naturelles. ${ }^{83}$

Incontestablement, les incriminations spécifiques au Protocole de Malabo visent des faits qui font des ravages en Afrique, voire globalement, quoiqu'ils ne figurent pas dans le Statut de Rome en tant que crimes qui « touchent l'ensemble de la communauté internationale ». Ademola Abass est d'avis que le fait même de cette omission justifie en soi les spécificités du Protocole de Malabo ${ }^{84}$.

Parmi ces nouvelles incriminations internationales en Afrique, le crime qui touche aux actes constitutifs de changement anticonstitutionnel de gouvernement (CACG) revêt un intérêt régional particulier à bien d'égards. Tout d'abord, le changement inconstitutionnel de gouvernement constitue une préoccupation historique de l'UA et de l'Organisation de l'unité africaine (OUA) ${ }^{85}$. Ensuite et plus intéressant encore, les pratiques que le Protocole de Malabo réprime sous ce label vont au-delà des formes classiques de renversement de gouvernement par voie de coup d'État militaire ${ }^{86}$, de rébellion ${ }^{87}$ ou encore par recours à des mercenaires ${ }^{88}$. Le crime couvre toute une gamme d'actes illégaux de maintien des dirigeants africains au pouvoir, lesquels constituent une pandémie sur le continent dans son ensemble. Les actes ainsi visés sont: le refus des dirigeants en place de céder le pouvoir au vainqueur à l'issue des élections; des amendements illégaux des constitutions en violation des principes d'alternance démocratique au pouvoir; des modifications unilatérales et « spéculatives » des lois électorales ${ }^{89}$.

Davantage que les coups d'État militaires classiques, en effet, la gestion des crises liées aux pratiques contemporaines en Afrique qualifiées par Ahmedou Ould-

83 Protocole de Malabo, supra note 71.

84 Voir Ademola Abass, «The Proposed International Criminal Jurisdiction for the African Court: Some Problematical Aspects » (2013) 60:1 Nethl Int'l L Rev 27 à la p 34 [Abass, « Problematical Aspects »].

85 Adoptée le 30 juin 2007, la Charte Africaine de la Démocratie, des Élections et de la Gouvernance (CADEG) est le premier instrument de nature conventionnelle à définir et condamner le crime en Afrique. Les préambules de la Charte reprennent l'historique des décisions, résolutions et documents d'orientation politique condamnant les pratiques de changement anticonstitutionnel de gouvernement : la Déclaration sur la situation politique et socio-économique en Afrique et les changements fondamentaux qui se produisent dans le monde, AHG/Dec. 11 (XXVI), 1990, adoptée lors du 26e sommet de l'OUA, Addis Abeba, 9 au 11 juillet 1990; l'Agenda du Caire de 1995 pour la relance économique et le développement social en Afrique, la Décision d'Alger de 1999 sur les changements anticonstitutionnels de gouvernement, la Déclaration de Lomé de 2000 sur une réaction de 1'OUA face aux changements anticonstitutionnels de gouvernement, la Déclaration de l'OUA/UA sur les principes régissant les élections démocratiques en Afrique adoptée en 2002, le Protocole de 2003 portant création du Conseil de Paix et de Sécurité de l'Union africaine. Pour une exploration détaillée de l'historique du crime d'une condamnation dans la pratique des États jusqu'à cristallisation et sa consécration conventionnelle, voir Abass, «International Crimes », supra note 73 aux pp 939-941.

86 Protocole portant amendements au Protocole portant Statut de la Cour africaine de justice et des droits de l'homme, 27 janvier 2015, article 28E(1)a).

87 Ibid, art $28 \mathrm{E}(\mathrm{c})$.

88 Ibid, art 28E(b).

89 Ibid, art 28E(d). 
Abdallah, ancien envoyé spécial du Secrétaire général de l'ONU Boutros-Boutros Ghali au Burundi, de «coups d'État civil» représente un des plus grands défis contemporains de l'UA ${ }^{90}$. La question peut se poser d'ailleurs de savoir si ceux-ci ne risquent pas de rompre le consensus au sein de l'opinion publique sur la condamnation des coups d'État militaires classiques ${ }^{91}$. Il est donc tout à fait logique qu'un traité régional de droit pénal international en Afrique incrimine les actes constitutifs du crime de CACG. Point n'est besoin de démontrer l'intérêt de la répression de cette criminalité dans le cas du Burundi, lequel est nommément cité par les experts comme l'un des exemples de ce type de comportement des dirigeants en place ${ }^{92}$.

La pratique prometteuse de l'EACJ dans les domaines de la gouvernance, l'État de droit et les droits de l'homme milite en faveur d'un organe de droit pénal international régional au sein des structures actuelles de l'EAC. D'emblée, il faut noter que l'EACJ n'est pas dotée d'une compétence explicite dans ces matières. En effet, le premier alinéa de l'article 27 du Traité de l'EAC dispose que l'EACJ est « initialement » compétente en matière d' « interprétation et d'application du Traité », tandis que le deuxième alinéa de cette disposition prévoit l'éventualité d'une extension des compétences de l'EACJ aux questions touchant les droits de l'homme ${ }^{93}$.

Le sommet des chefs d'État, tenu le 30 novembre 2013 à Kampala en Ouganda, a considéré, mais sans entériner une proposition du Conseil des ministres des États membres de la Communauté (Conseil des ministres de l'EAC ou Conseil) d'étendre effectivement les compétences de la Cour en matière de droits de l'homme. À l'issue du sommet, les chefs d'États et de gouvernement ont plutôt recommandé au Conseil de mener des consultations préalables sur ce sujet avec l'UA ${ }^{94}$.

90 Ahmedou Ould-Abdallah, « Après les coups d'État militaires, l’Union africaine à l'épreuve des coups d'État civils », Le Monde (17 juillet 2016), en ligne : Le Monde <www.lemonde.fr> (s'exprimant à la veille du sommet de l'UA tenu les 17 et 18 juillet 2016 à Kigali au Rwanda).

91 Ibid. Dans un langage qui semble implicitement décrire le cas du Burundi, Ahmedou Ould-Abdallah semble insinuer que, même au « risque des condamnations internationales », la population pourrait voir en un coup d'État militaire le seul moyen d'éviter une guerre civile face à « [...] un président au comportement erratique exécutant ses opposants, encourageant la violation des droits de l'homme et le népotisme comme base de recrutement ainsi que la corruption systématique comme mode de gouvernance et, surtout, se déclarant en contact direct avec Dieu [...] ». Dans tous les cas, des opinions dans ce sens ont été publiquement émises dans le contexte de la tentative de coup d'État militaire du 15 mai 2015 au Burundi. Voir par ex Jean Baptiste Placca, «La décence de ne pas s'en mêler » (16 mai 2005), en ligne : RFI <www.rfi.fr>.

92 Gerhard Werle et Moritz Vormbaum, «Creating an African Criminal Court», dans Gerhard Werle et Moritz Vormbaum, dir, The African Criminal Court: A commentary, International Criminal Justice Series, 10, Berlin: Humboldt-Universität zu Berlin, 2017 à la p 4.

93 Traité de l'EAC, supra note 64.

94 EAC, communiqué, «Communiqué of the 15th Ordinary Summit of the EAC Heads of State » (30 novembre 2013), en ligne: <http://repository.eac.int> au para 16 . 
Il est par ailleurs intéressant de noter que le Conseil des ministres avait en outre proposé de doter l'EAC d'une compétence en droit pénal international, quoique d'une portée limitée, puisqu'elle ne devait couvrir que les crimes contre l'humanité ${ }^{95}$. Des observateurs ont émis des doutes tout à fait fondés, par ailleurs, quant aux motivations réelles à la base de ce projet ${ }^{96}$. En effet, l'initiative est intervenue dans le contexte des poursuites pénales contre de hauts cadres du gouvernement kenyan, dont le président en exercice Uhuru Kenyatta ainsi que son vice-président, William Ruto, pour les crimes commis dans le cadre des violences postélectorales survenues en 2007 au Kenya ${ }^{97}$. Si le processus avait abouti, l'EACJ aurait été désormais compétente pour connaître des faits constitutifs de crimes contre l'humanité dont les suspects étaient poursuivis devant la CPI. L'étape suivante aurait alors été d'envoyer une requête à la CPI pour demander un transfert des dossiers afférents à ces actes à l'EACJ ${ }^{98}$. Cela dit, comme les critiques mêmes le suggèrent, les circonstances particulières qui entourent cette initiative ne remettent cependant pas en cause le bien-fondé même de la proposition $^{99}$.

En effet, la jurisprudence de l'EACJ démontre son engagement réel dans les domaines liés à l'État de droit dans les États membres de la Communauté. Plus intéressant encore, elle démontre une volonté de résistance des juges afin de défendre les principes de l'État de droit, y compris dans un contexte politique peu favorable. Au cours des dix dernières années, l'EACJ a adopté une interprétation dynamique et créative du Traité de l'EAC dans le traitement des questions liées à des violations des droits de l'homme, malgré les limites du texte et en dépit de la protestation persistante des États. De même, l'approche holistique adoptée par l'EACJ dans l'interprétation du Traité de l'EAC a permis aux juges de condamner les différents types de violation de droits, qu'il s'agisse de droits civils et politiques ou environnementaux ${ }^{100}$. Plus

95 Ibid.

96 Voir par ex Kai Ambos, «Introduction» dans Kai Ambos et Ottilia A Maunganidze, dir, Power and Prosecution: Challenges and Opportunities for International Criminal Justice in Sub-Saharan Africa, Göttingen, Universitätsverlag Göttingen, 2012 à la p 13 [Ambos, « Introduction »].

97 The Prosecutor v Francis Kirimi Muthaura, Uhuru Muigai Kenyatta and Mohammed Hussein Alim, ICC-01/09-02/11-1, Decision on the Prosecutor's Application for Summonses to Appear for Francis Kirimi Muthaura, Uhuru Muigai Kenyatta and Mohammed Hussein Ali (8 mars 2011) (CPI, Chambre préliminaire II); The Prosecutor v William Samoei Ruto, Henry Kiprono Kosgey and Joshua Arap Sang, ICC-01/09-01/11-373, Decision on the Prosecutor's Application for Summons to Appear for William Samoei Ruto, Henry Kiprono Kosgey and Joshua Arap Sang (2 février 2012) (CPI, Chambre préliminaire II) [Affaire Ruto, Kogsey et Sang].

98 Le document de base émanait du député Kenyan, Hon. Dan Wandogo Andera et a été endossé par l'East African Legislative Assembly (ici EALA). Voir EALA, Resolution of the Assembly seeking the EAC Council of Ministers to implore the International Criminal Court to transfer the case of the accused four Kenyans facing trial in respect of the aftermath of the 2007 Kenya general elections to the East African Court of Justice and to reinforce the treaty provision, 26 avril 2012, aux para 4-6, en ligne : <www.eala.org $>$.

99 Voir par exemple, Ambos, «Introduction », supra note 96, à la p 11.

100 Une affaire impliquant les droits environnementaux portait sur un projet de construction d'une autoroute qui devait traverser le Serengeti National Park par le gouvernement tanzanien. Voir African Network for Animal Welfare (ANAW) v Attorney General of the United Republic of Tanzania (Ref No 9, 2010) (EACJ). Pour une analyse intéressante situant l'affaire dans un contexte plus large de revendication judiciaire des droits environnementaux devant les mécanismes régionaux en Afrique, 
récemment, la Cour a même eu à débattre d'un cas inédit dans le cadre d'une procédure judiciaire supranationale, en l'occurrence la question du troisième mandat contesté du président Pierre Nkurunziza, touchant ainsi à des aspects du problème de changement inconstitutionnel de gouvernement évoqué ci-haut ${ }^{101}$.

Une première décision de principe de l'EACJ dans le domaine des droits de l'homme est intervenue en 2007 dans le cadre de l'affaire Katabazi, procès emblématique mettant en cause l'État de l'Ouganda ${ }^{102}$. Dans cette affaire, les requérants avaient été détenus en Ouganda pour actes de trahison et non-révélation de la haute trahison vers fin $2004^{103}$. Certains d'entre eux avaient alors demandé et obtenu l'octroi de mandats de libération moyennant caution par une haute juridiction ougandaise, la Haute Cour de l'Ouganda. Alors que les juges de la haute juridiction préparaient les documents de libération, un groupe paramilitaire gouvernemental était intervenu et avait procédé à l'arrestation des prévenus, dans l'enceinte même de la Cour, le 15 novembre $2005^{104}$. Les détenus avaient par la suite été conduits devant une juridiction militaire, qui les avait accusé de détention illégale d'armes à feu et de terrorisme pour les faits mêmes sur la base desquels ils avaient bénéficié des mandats de libération ${ }^{105}$. Ils ont été maintenus en détention en dépit d'une décision ultérieure de la Cour constitutionnelle ougandaise constatant de l'inconstitutionnalité des arrestations et ordonnant la libération des détenus ${ }^{106}$.

Devant l'EACJ, les requérants ont accusé l'Ouganda d'avoir agi ainsi en violation des articles 6, 7(2), et 8(1) du Traité de l'EAC. L'article 6 du Traité de l'EAC énonce le respect et la promotion des droits de l'homme en conformité avec la Charte Africaine des Droits de l'Homme et des Peuples ${ }^{107}$ parmi les principes fondamentaux qui lient les États parties dans l'accomplissement des objectifs de l'EAC ${ }^{108}$. L'article 7(2) du Traité de l'EAC complète les dispositions de l'article 6 en précisant les objectifs de la Communauté : «les principes de bonne gouvernance, y compris l'adhésion aux principes de la démocratie, l'État de droit, la justice sociale ainsi que le respect des standards universels des droits de l'homme ${ }^{109}$ [Notre

voir James Thuo Gathii, «Saving the Serengeti: Africa's New International Judicial Environmentalism » (2016) 16:2 Chi J Int'1 L 385.

101 The East African Civil Society Organizations' Forum (EACSOF) v The Attorney General of the

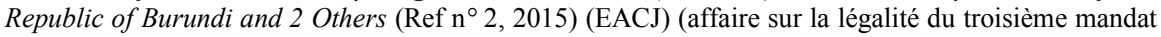
du president Nkurnziza) [EACSOF].

102 Katabazi et 21 autres c Le Secrétaire général de la Communauté d'Afrique de l'Est et l'"Attorney General" de la République d'Ouganda $\left(\operatorname{Ref}^{\circ}{ }^{\circ} 1,2007\right)$ (EACJ) [Katabazi].

103 Ibid à la p 1.

104 Ibid. L'EACJ utilise l'expression «security personnel». Par contre, les termes «paramilitary personnel » sont employés par le centre de développement constitutionnel au sein de l'EAC. Kituo Cha Katiba, «Five-Year Strategic Plan 2011-2016 », Eastern Africa Centre for Constitutional Development (KCK) (2010) à la p 10 [Katiba], cité dans James Thuo Gathii, «Variation in the Use of Subregional Integration Courts Between Business and Human Rights Actors: The Case of the East African Court of Justice » (2015) 79:1 Law \& Contemp Probs 37 à la p 55 [Gathii].

105 Katiba, ibid à la p 2.

106 Katabazi, supra note 102 à la p 2.

107 Charte africaine, supra note 74.

108 Traité de l'EAC, supra note 64, art 6(d).

109 Ibid, art 7(2). 
traduction et nos italiques]. Enfin, l'article 8(1)(c) du Traité de l'EAC oblige les États parties à s'abstenir de toute mesure susceptible d'entraver la réalisation de ces objectifs ou de la mise en œuvre des dispositions du Traité de l'EAC ${ }^{110}$.

Sans surprise, l'Ouganda a soulevé comme principal moyen de défense l'exception d'incompétence de l'EACJ dans les matières relatives aux droits de l'homme ${ }^{111}$. Les juges de l'EACJ ont cependant rejeté les arguments des autorités ougandaises critiquant, par la même occasion, les agissements de l'État ougandais comme constituant « un précédent inacceptable et dangereux susceptible de saper les fondements de l'État de droit $\gg{ }^{112}$ [Notre traduction]. La Cour a maintenu sa ligne de raisonnement dans une série de décisions ultérieures relatives à des questions concernant l'État de droit et les droits de l'homme ${ }^{113}$. La Division d'appel de l'EACJ a eu l'occasion de confirmer la position de la Cour qui avait été exprimée sur un ton on ne peut plus ferme en première instance dans l'affaire Rugumba contre Rwanda. La décision de première instance confirmée en appel lisait notamment:

[...] we are of the firm view that the principles set out in Article 6(d) and 7(2) were not inscribed in vain. The Jurisdiction of this Court to interpret any breach of those Articles was also not in vain, neither was it cosmetic. The invocation of the provisions of the African Charter on Human and Peoples Rights was not merely decorative of the Treaty but was meant to bind Partner States hence the words that Partner States must bind themselves to the "adherence to the principles of democracy, the rule of Law [...] as well as the recognition, promotion and protection of Human and Peoples Rights in accordance with the provisions of the African Charter on Human and Peoples Rights" (ACHPR). ${ }^{114}$

De l'avis de la Cour, la détention incommunicado d'un citoyen rwandais durant cinq mois sans jugement et sans que le détenu ait pu avoir accès à un juge compétent constituait une violation des principes de bonne gouvernance et de l'État de droit, principes consacrés par les articles 6(d) et 7(2) du Traité de l'EAC ${ }^{115}$.

Pour revenir sur la question du troisième mandat du Président Nkurunziza, il faudrait noter que l'affaire revêtait un intérêt très particulier pour les individus et les groupes de défenses des principes de l'État de droit en Afrique, qui n'est pas nécessairement lié à l'issue de l'affaire. De fait, elle a été l'occasion d'introduire, pour la première fois, un débat judiciaire sur la violation des limitations constitutionnelles des mandats présidentiels en Afrique devant une juridiction supranationale ${ }^{116}$. L'on

110 Ibid, art 8(1)(c).

111 Katabazi, supra note 102 à la p 12.

112 Ibid à la p 22.

113 James Thuo Gathii note que l'EACJ a déjà été saisie d'au moins soixante affaires portant sur des questions relatives aux droits de l'homme. Gathii, supra note 104 à la p 38.

114 Plaxeda Rugumba $v$ The Secretary General of the East African Community and The Attorney general of the Republic of Rwanda (Ref $\left.\mathrm{n}^{\circ} 8,2010\right)$ (EACJ) au para 37 (Pour la décision en appel, voir The Attorney General of the Republic of Rwanda c Plaxeda Rugumba, (Appeal n 1, 2012) (EACJ)).

115 Ibid au para 42.

116 Dans ces termes, voir Pan-African Lawyers' Union, Communiqué, « Public Hearing of Reference No. 2 of 2015: East African Civil Society Organizations' Forum (EACSOF) vs. The Attorney General 
comprend ainsi que l'affaire ait mobilisé la société civile aux niveaux local, sous régional et panafricain, qui lui attribuaient une «importance historique ${ }^{117}$.

L'affaire a été introduite le 6 juillet 2015 par l'East African Civil Society Organisations' Forum (EACSOF), une plate-forme régionale d'ONGs émanant de la société civile, représentée par la Pan African Lawyers' Union (PALU) ${ }^{118}$. Les requérants ont attaqué une série de mesures en rapport avec le processus électoral de 2015 au Burundi à savoir : la nomination du président Nkurunziza comme candidat par son parti politique le CNDD-FDD, puis son élection aux présidentielles contestées de 2015. Ils soutenaient que ces décisions, de même que l'arrêt polémique $R C C B 303^{119}$ de la Cour constitutionnelle du Burundi validant la candidature du président Nkurunziza, violaient «la lettre, l'esprit et les objectifs » de l'Accord d'Arusha ${ }^{120}$ et de la Constitution du Burundi ${ }^{121}$.

Le Burundi était accusé d'avoir enfreint une série de dispositions du Traité de l'EAC relatives aux obligations de promotion de la paix, de la sécurité, de la stabilité au sein de la communauté, ainsi que de maintien des rapports de bon voisinage parmi les pays membres ${ }^{122}$; d'adhésion aux principes de bonne

of Burundi, the Commission électorale nationale indépendante (CENI) and the Secretary General of East African Community (EAC) » (10 juin 2016), en ligne : <lawyersofafrica.org >

117 Ibid.

118 EACSOF, supra note 101.

119 Arrêt RCCB 303, Cour constitutionnelle du Burundi, en ligne : <www.uantwerpen.be>.

120 Accord d'Arusha, supra note 17, art 7(3), Protocole II. « Il [le président de la République] est élu pour un mandat de cinq ans renouvelable une seule fois. Nul ne peut exercer plus de deux mandats présidentiels »).

121 L'article 96 de la Constitution limite le nombre de mandats présidentiels à deux : « [1]e Président de la République est élu au suffrage universel direct pour un mandat de cinq ans renouvelable une fois ». De son côté, l'article 302 al 1 apporte un tempérament à cette disposition en précisant qu' «à titre exceptionnel, le premier Président de la République de la période post-transition est élu par l'Assemblée Nationale et le Sénat élus réunis en Congrès, à la majorité des deux tiers des membres. " C'est complètement à tort que la Cour constitutionnelle du Burundi s'est largement basée sur cette disposition pour valider un troisième mandat du président Nkurunziza par un arrêt très controversé et polémique, rendu le 5 mai 2015 au lendemain de l'exil du vice-président de la Cour constitutionnelle qui dénonçait des menaces sur les juges ayant siégé dans l'affaire. Comme le professeur Gervais Gatunange le démontre clairement, l'exception de l'article 302 al 1 «porte uniquement sur le mode d'élection et non sur le nombre de mandats ». Gervais Gatunange, "Analyse de la Constitution commanditée par le FOCODE : De la conformité à la constitution d'un autre mandat du Président Pierre Nkurunziza », (avril 2015), en ligne: FOCODE (Forum pour la Conscience et le Développement) < burundinews.fr/actualites/GATUNANGE3mandat.pdf $>$ aux pp 2-3. Dans le même sens, le professeur Pacifique Manirakiza explique la portée de cette exception dans son contexte historique. Voir Pacifique Manirakiza, «The 2005 Constitution of Burundi », University of Pretoria, Institute for International and Comparative Law in Africa (ICLA), en ligne: ICLA $<$ www.icla.up.ac.za> aux pp 20-22, Busingye Kabumba critique également un arrêt constitutionnel en violation d'un des principes cardinaux en matière d'interprétation des dispositions constitutionnelles : «[o]ne of the cardinal rules of constitutional interpretation is that a constitution must be read as an integrated whole, to ascertain its broad purpose and meaning, rather than in a piecemeal fashion which might lead to a result which is inimical to its broader architecture ») Busingye Kabumba, « The Provision that Could Break a Nation: Article 96 of the Burundi Constitution » (2015), en ligne : Uganda Journalist Resource Center <www.ugandajournalistsresourcecentre.com $>$ à la $\mathrm{p} 3$.

122 Traité de l'EAC, supra note 64, art 5(3)(f) («[f]or purposes set out in paragraph 1 of this Article and as subsequently provided in particular provisions of this Treaty, the Community shall ensure: [...] the 
gouvernance, de démocratie, d'État de droit, de respect et de promotion des droits de l'homme en vertu des articles $6(d)$ et $7(2)^{123}$. Les requérants avaient saisi la Cour à la veille des élections présidentielles et sénatoriales de juin 2015 en requête d'une décision ordonnant le report desdites élections au titre des mesures provisoires et conservatoires en attendant l'issue des débats au fond, mais n'avaient pas eu gain de gain cause sur ce point ${ }^{124}$. Une session de débats sur le fond a eu lieu en audience publique le 13 juin 2016. Dans la décision rendue le 29 septembre 2016 par la première division de l'EACJ, la Cour s'est déclarée incompétente en l'espèce ${ }^{125}$. Une décision qui n'enlève rien cependant à l'intérêt de notre proposition.

L'implication de la société civile dans le fonctionnement de la Cour constitue justement un autre aspect intéressant. En effet, l'intervention constante de l'EACJ dans les domaines liés à la gouvernance, l'État de droit et les droits de l'homme en dépit de la résistance persistante des États a été rendue possible, dans une large mesure, par une implication significative des ONG et des groupes de défense des droits dans le fonctionnement de la Cour légitimant, en quelque sorte, ses interventions. À ce propos, James Thuo Gathii décrit en détail le rôle de l'East African Law Society, une plate-forme d'avocats membres des barreaux de la sousrégion ${ }^{126}$, une participation de la société civile qui a été, à son tour, rendue possible par l'ouverture et la flexibilité des juges à cet égard ${ }^{127}$. Or, une Cour ouverte à la participation de la société civile dans son fonctionnement est susceptible de mieux protéger les droits des victimes.

\section{Approche d'une complémentarité positive en cas de conflits de compétences entre juridictions supranationales}

Le débat sur la complémentarité entre les instances étatiques et la CPI a toujours suscité un grand intérêt dans la littérature relative à l'administration de la justice pénale internationale sous l'empire du Statut de Rome ${ }^{128}$. Initialement, cet intérêt se justifiait par la place centrale qu'occupent les dispositions réglant la

promotion of peace, security and stability within, and good neighbourliness among, the Partner States »)

123 Les autres dispositions invoquées sont les articles 8(1)(a) et (c) (obligations de mise en œuvre des dispositions du Traité et de s'abstenir de toute mesure qui en entraverait la réalisation), ainsi que 8(5) (obligation d'assurer la primauté du droit communautaire sur les lois nationales). Pour un résumé de la requête, voir EACJ, «First Instance Division dismiss a case challenging the decision of the Constitutional Court of Burundi on the Presidential term limit » ( 30 septembre 2016), en ligne : EACJ $<$ www.eacj.org/?p=3251 > [EACJ, « First Instance »].

124 East Africa Civil Society Organisations' Forum (EACSOF) v Attorney General of Burundi, Commission electorale nationale independente (CENI) and Secretary General, East African Community (App n ${ }^{\circ}$, 2015) (EACJ).

125 EACJ, « First Instance », supra note 123.

126 Gathii, supra note 104 à la p 54.

127 Ibid à la p 37.

128 Voir généralement Jon Holmes, «Complementarity: National Courts versus the ICC » dans Antonio Cassese, Paola Gaeta et John RWD Jones, dir. The Rome Statute of the International Criminal Court: A Commentary, Oxford, Oxford University Press, 2002, 667 [Holmes]. 
coopération ainsi que la gestion des conflits de compétence entre les États et la CPI dans la poursuite et la répression des crimes relevant du Statut de Rome. Par la suite, les tensions entrainées par l'application effective de ces dispositions ont suscité un regain d'intérêt sur la question ${ }^{129}$.

Les projets de création des mécanismes de droit pénal international en Afrique ajoutent une dimension toute nouvelle aux débats. Jusqu'ici, la complémentarité était envisagée uniquement entre les instances étatiques et la CPI. Ce qui était logique puisque le régime de l'article 17 Statut de Rome prévoit un seul ordre de complémentarité à savoir national-international (CPI). Par contre, le Protocole de Malabo envisage deux niveaux de compétence supplémentaires à l'échelle supranationale: sous-régional dans le cadre des CER, et continental au sein des structures de l'UA. Il faut dorénavant entrevoir la gestion des conflits de compétences entre non plus seulement des instances nationales et une juridiction internationale, mais également entre des juridictions supranationales. Concrètement, il faut envisager les paliers suivants: national, sous-régional, continental (Africain), international (CPI), du moins pour un État qui serait partie aux deux instruments, comme cela pourrait être le cas du Burundi.

Dans le souci d'une politique de complémentarité positive dans ce contexte, la proximité géographique de la juridiction supranationale du territoire de l'État sur lequel les crimes ont été commis est proposée par cet auteur comme critère de principe pour déterminer la juridiction qui devrait bénéficier d'une priorité de poursuites. Un aperçu comparé des régimes de complémentarité sous le Statut de Rome et le Protocole de Malabo introduit le propos.

\section{A. Analyse comparée du principe de complémentarité dans le Statut de Rome et le Protocole de Malabo}

1. NOTION ET RÉGIME DE COMPLÉMENTARITÉ DANS LE STATUT DE ROME

L'article 1 du Statut de Rome pose le principe selon lequel «la CPI est complémentaire des juridictions pénales nationales $»^{130}$. Le principe de la subsidiarité de la CPI par rapport aux juridictions nationales en soi ne suscite pas de débats. Audelà du fait qu'il permet de préserver la souveraineté et les intérêts des États dans la répression des crimes relevant de leur compétence, la règle repose sur des considérations communément admises liées à une bonne administration de la justice pénale internationale, à savoir : un accès aux éléments de preuve plus facile pour les États que pour la CPI, les coûts nettement plus raisonnables des procès nationaux, etc. Selon les termes du document de politique du Bureau du procureur de la CPI de septembre 2003, le régime de la complémentarité dans le Statut de Rome, en plus de traduire la volonté des États parties de se réserver la priorité des poursuites, se fonde sur des considérations d'《efficacité » et d'«effectivité » de la justice pénale

129 Pour une analyse synthétique et critique intéressante, voir Kevin Jon Heller, « Radical Complementarity » (2016) 14:3 J Int'l Criminal Justice 637 [Heller, « Radical Complementarity »].

130 Statut de Rome, supra note 1. 
internationale ${ }^{131}$. Enfin, la justice pénale internationale répond mieux aux besoins des communautés touchées par les crimes si elle est administrée à proximité des lieux de commission des crimes ${ }^{132}$.

Par conséquent, en application des règles sur la complémentarité, lorsqu'un État compétent et la CPI (le Bureau du procureur) se disputent la conduite des poursuites dans une «même affaire », l'État l'emporte en principe. Le premier paragraphe de l'article 17 du Statut de Rome précise les modalités de mise en œuvre du principe en énumérant tour à tour quatre situations dans lesquelles la CPI devra déclarer ainsi l'irrecevabilité d'une affaire aux motifs que l'affaire en cause fait l'objet d'enquête ou de poursuites au niveau étatique, ainsi que les exceptions au principe ${ }^{133}$. La première de ces quatre exceptions nous intéresse particulièrement ici. Elle est posée à l'article 17(1)(a) du Statut de Rome ${ }^{134}$. Selon cette disposition, une affaire est jugée irrecevable par la Cour lorsque "l'affaire fait l'objet d'une enquête ou de poursuites de la part d'un État ayant compétence en l'espèce, à moins que cet État n'ait pas la volonté ou soit dans l'incapacité de mener véritablement à bien l'enquête ou les poursuites $\gg^{135}$.

Dans le cadre des débats sur les exceptions d'irrecevabilité au motif que l'affaire poursuivie par la CPI « fait l'objet d'une enquête ou de poursuites » par les autorités judiciaires de l'État compétent, la CPI (Chambre préliminaire) vérifie d'abord, selon un certain nombre de critères, si les poursuites nationales concernent effectivement la même affaire. Dans l'affirmative, la Cour passe alors aux volets suivants du test : celui du " manque de volonté » d'abord; celui lié à l' " (in)capacité de mener véritablement à bien l'enquête ou les poursuites" en vue de traduire le suspect devant la justice ensuite. Sous le volet «manque de volonté »-formulé de façon négative -, le paragraphe 2 de l'article 17 du Statut de Rome prévoit que la Cour pourra conclure dans ce sens, si elle estime que l'enquête ou les poursuites engagées au niveau national visent plutôt à soustraire la personne poursuivie de la justice ${ }^{136}$. Un tel dessein pourra se déduire des circonstances telles «qu'un retard injustifié » ou le manque d' " indépendance ou impartialité » dans la conduite des procédures nationales ${ }^{137}$. S'agissant de l'incapacité de poursuivre, celle-ci doit découler « de l'effondrement de la totalité ou d'une partie substantielle (de l'appareil judiciaire de l'État concerné) ou de l'indisponibilité de celui-ci » ${ }^{138}$.

La signification et la portée des expressions «manque de volonté et/ou incapacité de mener véritablement à bien des poursuites » employées dans la formulation de l'article 17 au regard des objectifs visés par les dispositions de celui-ci

131 ICC, Office of the Prosecutor, «Paper on Some Policy Issues Before the Office of the Prosecutor » (septembre 2003), en ligne : ICC <www.icc-cpi.int> à la p 2.

132 Voir en ce sens Neil Kritz, "Progress and Humility: The Ongoing Search for Post-Conflict Justice » dans M Cherif Bassiouni, dir, Post-Conflict Justice, Ardsley, Transnational Publishers, 2002, 55.

133 Statut de Rome, supra note 1, art 17.

134 Ibid, art 17(1)(a).

135 Ibid.

136 Ibid, art 17(2)(a).

137 Ibid, art 17(2)(b).

138 Au sujet d'une analyse sur la portée de ces expressions, voir Holmes, supra note 128. 
ont fait l'objet d'intenses débats et de controverses dans la littérature ${ }^{139}$. La CPI a, pour sa part, eu l'occasion de préciser l'interprétation qu'elle donne à ces dispositions dans le cadre des débats sur la recevabilité des affaires visant les situations au Kenya, puis en Libye ${ }^{140}$.

\section{LE RÉGIME DE COMPLÉMENTARITÉ DANS LE PROTOCOLE DE MALABO}

Le Protocole de Malabo reprend les dispositions du Statut de Rome s'agissant de la complémentarité de la SDPI par rapport aux instances étatiques. Sur cet aspect, le deuxième paragraphe de l'article $46 \mathrm{H}$ du Protocole de Malabo est presque l'équivalent du premier paragraphe de l'article 17 du Statut de Rome. En revanche, la spécificité du Protocole de Malabo réside dans le fait qu'il prévoit, en plus de la complémentarité de la SDPI aux instances étatiques, l'éventualité d'une subsidiarité de la juridiction continentale à un mécanisme de droit pénal international sous-régional préexistant au Protocole de Malabo ou qui serait ultérieurement établi au sein d'une CER et qui serait concurremment compétent pour mener des poursuites dans l'État visé. Aux termes de l'article $46 \mathrm{H}$, paragraphe premier du Protocole de Malabo, «la juridiction de la Cour est complémentaire à celle des juridictions nationales et éventuellement à celle des Communautés économiques régionales quand cela est expressément prévu par lesdites communautés $\gg^{141}$ [Nos italiques].

Ademola Abass relève certaines préoccupations d'ordre pratique au sujet de cette innovation africaine en matière de complémentarité ${ }^{142}$. La première découlerait du fait que des États africains sont simultanément parties à plus d'une organisation sous-régionale, ce qui est notamment le cas du Burundi. L'auteur s'interroge alors sur le fait de savoir lequel de ces mécanismes devrait être pris en considération aux fins de la complémentarité dans le cas d'un État membre de plus d'une CER, instituant chacune un mécanisme de droit pénal international ${ }^{143}$. La deuxième préoccupation de l'auteur tient au fait que les règles de procédures applicables devant certaines instances régionales créent des obstacles quant à l'accès des individus à ces systèmes (la Cour africaine des droits de l'homme et des peuples est spécifiquement visée). L'auteur s'inquiète alors quant à la légitimité de considérer, dans le cadre de la complémentarité, la priorité d'intervention de ce genre de mécanismes ${ }^{144}$.

139 Pour une synthèse des courants doctrinaux en présence ainsi qu'une analyse particulièrement convaincante, voir Heller, « Theory to Complementarity », supra note 47.

140 Affaire Ruto, Kogsey et Sang, supra note 97 ; Decision on the Application by the Government of Kenya Challenging the Admissibility of the Case Pursuant to Article 19(2)(b) of the Statute; Le Procureur c Saif Al-Islam Gaddafi et Abdullah Al-Senoussi, ICC-01/11-01/11, Arrêt relatif à l'appel interjeté par la défense de M. Al-Senoussi de la décision de la Chambre préliminaire intitulée « Décision relative à la recevabilité de l'affaire à l'encontre d'Abdullah Al-Senoussi », rendue le 11 octobre 2013 (24 juillet 2014) [Affaire Saif Al-Islam Gaddafi et Abdullah Al-Senoussi]

141 Protocole de Malabo, supra note 71, art 46H.

142 Abass, « International Crimes », supra note 74 aux pp 944-945.

143 Ibid.

144 Ibid. 
Ces préoccupations sont fondées, mais il nous semble qu'elles portent essentiellement sur des questions d'ordre pratique. Elles ne remettent pas en question les fondements mêmes du principe de complémentarité du Protocole de Malabo qui, à défaut de poursuites nationales, reconnaît la priorité d'intervention à une juridiction sous-régionale par rapport à la SDPI envisagée au niveau continental. En règle générale, cette solution nous parait conforme à la logique d'une politique de complémentarité positive entre des juridictions supranationales. Il s'agit de l'approche justement avancée par cet article. Elle consiste à régler ce genre de conflit de compétence en accordant la primauté de compétence, en règle générale, à la juridiction internationale ayant la plus grande proximité géographique avec lieux des crimes, par hypothèse la juridiction sous-régionale.

Au reste, la question soulevée à propos des conflits de compétence entre des juridictions, toutes sous-régionales, se règlerait idéalement en amont dans le cadre de l'adhésion des États aux traités. À défaut, le critère même de la proximité géographique permettrait de trancher. En effet, même si les deux juridictions concurremment compétentes étaient toutes établies au sein des CER et par-là-même situées aux échelons de juridictions (sous-régionaux), l'on peut anticiper qu'il serait rare qu'elles soient situées à la même distance géographique du territoire de l'État en cause. Par exemple, le Burundi est à la fois partie de l'EAC et de la Communauté économique des États de l'Afrique centrale (CEEAC). Cela étant, un mécanisme de droit pénal international qui serait créé au sein de la CEEAC et établi, supposons, à Libreville (Gabon), siège actuel de la CEEAC, ne remplirait pas nécessairement les critères de proximité géographique de l'EACJ sise à Arusha en Tanzanie. L'EAC demeurerait géographiquement plus proche des populations burundaises, cette proximité physique s'accompagnant d'un attachement psychologique en raison de l'histoire régionale.

La deuxième question, celle qui a trait à l'opportunité d'accorder une priorité de poursuites à des juridictions sous-régionales alors même que les règles de procédures devant celles-ci entraveraient la justice, trouverait probablement une solution dans le cadre d'une jurisprudence de la SDPI, lorsque celle-ci sera opérationnelle. À cet égard, la jurisprudence de la CPI dans le cadre des débats sur les exceptions de recevabilité fournirait des sources d'inspiration utiles dans la mesure où, comme nous l'avons mentionné, les dispositions relatives au principe de complémentarité ainsi que les exceptions relatives figurant dans le Protocole de Malabo sont presqu'un copié-collé des règles du Statut de Rome, du moins s'agissant des rapports avec les États. Or, la CPI a établi une jurisprudence intéressante qui fait fixant l'objectif ultime d' "amener le suspect devant la justice », un critère pivot d'appréciation du "manque de volonté et/ou l'incapacité» [nos italiques] de poursuivre de la part d'un État qui prétend préserver la priorité de poursuites dans le cadre de l'article $17 \mathrm{du}$ Statut de Rome ${ }^{145}$.

Ainsi, les mêmes critères qui s'appliquent pour apprécier dans quelle mesure les poursuites devant les instances étatiques traduisent une volonté réelle et

145 Affaire Saif Al-Islam Gaddafi et Abdullah Al-Senoussi, supra note 140. 
démontrent la capacité de l'État de poursuivre, serviraient mutatis mutandis aux fins de la complémentarité entre la SDPI et les mécanismes sous-régionaux. À cet égard, l'évaluation porte autant sur la pertinence de la législation nationale qui forme le cadre légal des poursuites que sur les éléments de faits relatifs aux poursuites prétendues ou envisagées par l'État. Un cadre légal national inadéquat ou ineffectif traduirait un manque de volonté et/ou une incapacité de mener à bien des poursuites pénales. Dans la même optique, l'organe judiciaire d'une CER régi par un traité inadéquat ou ineffectif au point de compromettre les objectifs de la justice pénale internationale équivaudrait à une incapacité de la juridiction sous-régionale de mener à bien les poursuites. Celle-ci perdrait alors le privilège de l'article $48(\mathrm{H})$, paragraphe premier. En d'autres termes, pour préserver la primauté de poursuites, les mécanismes sous-régionaux devraient répondre à des critères de satisfaction à tout le moins comparables à ceux imposés aux instances étatiques.

Notons en passant la préférence, par les rédacteurs du Protocole de Malabo, des termes « réticence ou incapacité à réellement mener des poursuites » en lieu et place des expressions «manque de volonté ou incapacité de mener véritablement à bien des poursuites » employées dans le Statut de Rome. Le choix des termes n'est peut-être pas neutre de considérations idéologiques ou politiques, mais cela relève d'un autre débat ${ }^{146}$. Il est encore trop tôt pour spéculer sur les implications pratiques de ces différences de formulation entre les deux textes.

\section{B. La proximité géographique comme critère d'une primauté de compétence dans le cadre d'une complémentarité positive entre des juridictions supranationales}

1. NOTION ET APPROCHE D'UNE COMPLÉMENTARITÉ POSITIVE DANS LE CADRE DU STATUT DE ROME

L'expression «complémentarité positive » est couramment employée pour désigner une certaine politique d'intervention de la CPI. Elle a trait aux rapports de coopération entre la CPI et les États parties dans l'administration de la justice pénale internationale. Elle ne concerne pas nécessairement ni principalement l'interprétation et l'application technique des critères de recevabilité de l'article $17 \mathrm{du}$ Statut de Rome. L'idée renvoie beaucoup plus à une politique d'intervention du Bureau du procureur de la CPI qui valoriserait et encouragerait les poursuites nationales.

146 L'expression « manque de volonté et/ou incapacité » est critiquée par certains pour avoir une certaine connotation impérialiste. Voir à ce sujet Ntina Tzouvala, « Symposium on TWAIL Perspectives on ICL, IHL, and Intervention: TWAIL and the "Unwilling or Unable" Doctrine, Continuities, and Ruptures » (2016) 109 AJIL Unbound 266. Dans le cadre de la rédaction du Statut de Rome, l'expression anglaise "genuinely prosecute» traduite par «mener véritablement à bien des poursuites » dans la version française a été préférée à « effectively prosecute » considérée comme trop subjective par certaines délégations. Holmes, supra note 128. Quoique largement acceptée, l'expression «genuinely prosecute» n'a pas non plus fait l'unanimité. Paolo Benvenuti, «Complementarity of the International Criminal Court to National Criminal Jurisdiction » dans Flavia Lattanzi and William Schabas, dir, Essays on the Rome Statute of the International Criminal Court, vol I, Ripa Fagnano Alto, Il Sirente, 1999 à la p 42. 
L'ancien procureur de la CPI Louis Moreno-Ocampo l'évoquait ainsi implicitement :

$[a s]$ a consequence of complementarity, the number of cases that reach the Court should not be a measure of its efficiency. On the contrary, the absence of trials before this Court, as a consequence of the regular functioning of national institutions, would be a major success. ${ }^{147}$

Une politique de complémentarité positive a pour corollaire la possibilité pour les États en déficit de capacité de poursuites de pouvoir compter sur une assistance internationale sous forme de renforcement de ces capacités, du moins si les instances étatiques manifestent la volonté politique réelle de réprimer les crimes en application du Statut de Rome. Le rapport final du Bureau de l'Assemblée des États parties au Statut de Rome, tenue à Kampala en 2010, le rappelle à travers un document de politique adopté à l'issue de l'Assemblée :

[...] la complémentarité positive fait référence à toutes formes d'activités par lesquelles les capacités des instances judiciaires nationales sont renforcées et celles-ci sont capables de mener à bien les poursuites et la répression des crimes prévus sous le régime du Statut de Rome [...]. ${ }^{148}$ [Notre traduction]

Toutefois, la proposition faite par les dirigeants africains d'assigner à la CPI cette fonction d'assistance technique aux États a été rejetée ${ }^{149}$. Le bureau de l'Assemblée a renvoyé le traitement de cette question dans le cadre des relations bilatérales entre États ${ }^{150}$. L'intervention de la CPI en ce sens n'est donc pas à l'agenda de la Cour. Pour notre part, nous avons déjà mis en doute les capacités d'une juridiction globale à assumer cette fonction même si la volonté politique existe réellement. JURIDICTIONS SUPRANATIONALES

L'objectif ici est de déterminer laquelle des juridictions toutes supranationales concurremment compétentes, mais siégeant dans des espaces géographiques différents par rapport à l'État où les crimes ont été commis, devrait bénéficier d'une priorité d'intervention dans le cadre d'une politique d'intervention pénale internationale effective. Puisque le renforcement des capacités locales demeure au centre de toute politique de complémentarité positive, il est plus logique que la juridiction supranationale qui offre le plus grand potentiel dans la réalisation de cet objectif doive, si elle pouvait engager des poursuites, bénéficier d'une priorité de

147 Voir le discours de l'ancien procureur de la CPI, Louis Moreno-Ocampo, devant les corps diplomatiques. Luis Moreno-Ocampo, Prosecutor of the ICC, «Statement of the Prosecutor to the Diplomatic Corps » (12 février 2004), en ligne : <www.icc-cpi.int>.

148 ICC, Assembly of States Parties, « Report of the Bureau on Stocktaking: Complementarity. Taking Stock of the Principle of Complementarity : Bridging the Impunity Gap », ICC-ASP/8/51 (2010), en ligne : ICC <www.asp.icc-cpi.int $>$ au para 16.

149 Ibid.

150 Ibid. 
compétence. En vertu des arguments développés dans la deuxième partie de cet article, il s'agit donc de celle ayant la plus grande proximité physique avec le territoire de l'État où les crimes ont été commis.

En effet, les chances que la justice pénale internationale atteigne globalement ses objectifs y compris le renforcement des capacités locales, augmentent, en principe, avec la plus grande proximité de la juridiction qui poursuit avec les lieux où les crimes ont été commis. Inversement, ce potentiel diminue avec l'éloignement géographique de la juridiction. Cela dit, l'auteur admet le fait que la juridiction ayant la plus grande proximité géographique ne serait pas toujours forcément celle qui assure au mieux les objectifs de la justice post-conflit. La juridiction n'assumerait par exemple, la fonction réconciliatrice de la justice transitionnelle qu'à la condition que les règles d'organisation et de fonctionnement prévoient, notamment, le rôle et la place des victimes dans les procédures pénales. La solution que nous proposons en est une de principe et trouve ses fondements dans les arguments développés par cet article. Toutefois, elle peut souffrir des exceptions. Il n'y aurait pas de raison légitime à faire prévaloir la règle si la juridiction géographiquement la plus proche n'offre pas de garantie à cet égard. Tel serait le cas lorsque l'organisation et les modes de fonctionnement de la juridiction en question ne tiennent pas compte - ou insuffisamment - des besoins des victimes. La remarque rejoint en quelque sorte une des préoccupations d'Ademola Abass à laquelle l'auteur a tenté de répondre ${ }^{151}$.

À notre avis, c'est cependant ce critère qui pourrait donner une ligne d'interprétation de la solution proposée par cet article, celle retenue par l'article $46 \mathrm{H}$ du Protocole de Malabo qui reconnaît, comme nous l'avons relevé, la primauté de compétence aux instances sous-régionales par rapport à la SDPI ${ }^{152}$. Par ailleurs, un projet de document cadre d'une politique de l'UA en matière de justice transitionnelle en Afrique en fait une recommandation de politique générale sur le continent ${ }^{153}$. Il est également intéressant de noter que le document recommande une justice pénale centrée sur la participation des victimes ${ }^{154}$.

Encore une fois, l'opinion qui interprète la priorisation des institutions régionales africaines dans la répression des crimes internationaux commis sur le continent comme une sorte de confirmation de la préoccupation déjà évoquée selon laquelle les projets africains ne viseraient que l'établissement des «boucliers » contre l'intervention de la CPI en Afrique ${ }^{155}$ se comprend dans le contexte conjoncturel du projet. Sur le plan des principes, cependant, l'approche cadre mieux avec une bonne politique d'intervention pénale internationale dans les contextes de post-conflit (ou de conflits) qui prévalent en Afrique.

151 Abass, « Problematical Aspects », supra note 84 aux pp 39-40.

152 Protocole de Malabo, supra note 71 , art $46 \mathrm{H}$.

153 Union Africaine, «African Union Transitional Justice Framework », en ligne : Lawyer of Africa $<$ www.lawyersofafrica.org/wp-content/uploads/2014/11/AUTJF.pdf $>$ à la p 28 (version du document non finalisé) (« [...] an AUTJP [African Union Transitional Justice Policy] should seek to strengthen regional and domestic prosecutions of the most serious crimes $[\ldots] »)$.

154 Ibid à la p 12.

155 Mubiala, supra note 72 à la p 757. 
Bien entendu, une meilleure coopération entre la CPI et l'UA est souhaitable et reste la condition pour la réalisation de l'objectif communément partagé par les deux institutions, à savoir la prévention et la lutte contre l'impunité des crimes les plus graves. La réalité est que l'interdépendance entre les deux institutions semble inévitable dans cette entreprise. Du côté africain, la conscience de cette interdépendance transparaît à travers une série déclaration de politiques ainsi que des efforts orientés, semble-t-il, vers l'assainissement des relations entre l'UA et la CPI, allant jusqu'à proposer l'établissement de cadres formels de collaboration. Ainsi, le document sur la justice transitionnelle en Afrique susmentionné reconnaît que « l'entrée en vigueur du Statut de Rome et la création d'une toute première juridiction pénale internationale permanente fut une réalisation primordiale dans la lutte contre l'impunité des crimes internationaux ${ }^{156}$ [Notre traduction]. Par ailleurs, un rapport de 2013 du Groupe des sages de l'UA, produit dans le cadre de la mise en œuvre du document, déclare que «le document de politique marque le début d'une complémentarité positive entre l'Afrique et la justice internationale ${ }^{157}$ [Notre traduction]. Le rapport proposait la création d'un bureau de liaison entre la CPI et l'UA, qui assurerait « la mise en œuvre effective de la politique de complémentarité positive envisagée par le document ${ }^{158}$ [Notre traduction].

Que les déclarations traduisent une bonne volonté politique ou non, il n'y a pas de doute que l'intervention de la CPI en Afrique restera indispensable et sera accueillie favorablement parfois même par les détracteurs de la CPI. Il en sera au moins ainsi des dossiers impliquant des suspects présentant un certain profil, ce qui ne correspond évidemment pas à l'idéal d'une coopération dans la lutte contre l'impunité, bien que ce soit la réalité ${ }^{159}$.

Dans le contexte africain, on peut d'ailleurs toujours s'attendre à ce que la rhétorique contre l'intervention de la CPI en Afrique ne fasse pas l'unanimité parmi les dirigeants africains ${ }^{160}$. Le fait que la crise ait récemment atteint un nouveau pic avec le retrait d'États africains du Statut de Rome est indéniable. Nous ne pensons cependant pas que cela suffise pour présager d'une position commune et généralisée.

156 Union africaine, African Union Transitional Justice Framework, Addis-Abéba, 2014, cité dans Mubiala, supra note 72 à la p 757.

157 African Union Panel of the Wise, Peace, Justice, and Reconciliation in Africa: Opportunities and Challenges in the Fight Against Impunity, New York, International Peace Institute, 2013 à la p 81.

158 Ibid à la p 94.

159 C'est le cas d'anciens dirigeants ou chefs rebelles de très haut rang qui, même déchus ou en détention, garderaient des marges de pouvoirs et de soutiens populaires et dont les procès en Afrique emporteraient des risques de trouble. L'on peut s'imaginer les risques de déstabilisation politique qu'emporterait, par exemple, un procès contre l'ancien président ivoirien Laurent Gbagbo en Côte d'Ivoire, en Afrique de l'Ouest, voire au niveau continental. Pour une analyse critique de cette pratique qui consiste, pour les dirigeants africains à utiliser la CPI pour se débarrasser de leurs opposants, voir particulièrement José Alvarez qui s'exprime ainsi : «[t]he independence of the prosecutor and the Court can be undermined if states decide themselves to refer politically troublesome cases. An institution designed to be the last resort under complementarity is undermined if used as a convenient dumping ground for hot potato cases [...] ». José Alvarez, cité dans Philip Alston Ryan Goodman, dir, International Human Rights: The Successor to International Human Rights in Context, Oxford, Oxford University Press, 2013 aux pp 1351-1352.

160 Dans le même sens, voir par ex Ambos, supra note 55 à la p 510. 
Il faudrait d'ailleurs, et à ce propos, distinguer, y compris parmi les pays qui se sont effectivement retirés du Statut de Rome, les cas des États dont les motifs de retrait cadrent effectivement avec les raisons de protestation contre la CPI dans le contexte du mouvement de contestation plus ou moins généralisé sur le continent, de ceux qui ont peu en commun avec le mouvement. Le cas du Burundi, par exemple, loin de s'inscrire dans cette lignée se situe, au contraire, dans une logique d'auto-isolement entrepris au cours des dernières années, aussi bien sur la scène internationale que sur le continent africain ${ }^{161}$. Le cas de l'Afrique du Sud, par contre, repose sur la question controversée de l'opportunité des poursuites pénales contre de hauts dirigeants étatiques encore en exercice, objets de désaccords mêmes parmi les experts sur la justice pénale internationale en Afrique ${ }^{162}$.

Du côté de la CPI, l'importance d'une bonne coopération avec les États africains n'est pas à démontrer ${ }^{163}$. Des observateurs avisés estiment, d'ailleurs que les tensions entre la CPI et l'UA ne devraient pas être une fatalité dès lors que des efforts sont consentis des deux côtés ${ }^{164}$, et si la CPI elle-même adopte effectivement une meilleure approche de la complémentarité positive ${ }^{165}$.

\section{RISQUES DE FRAGMENTATION DU DROIT INTERNATIONAL ?}

La création d'un organe judiciaire régional comme modalité de régionalisation de la justice pénale internationale figurait déjà parmi les quatre propositions de William W Burke White dans le cadre de ce que l'auteur qualifiait d'« exploration préliminaire d'une mise en œuvre régionale de la justice pénale internationale $»^{166}$ [Notre traduction]. Cet auteur considérait cette forme de régionalisation qui consiste soit à créer des juridictions régionales de droit pénal international entièrement nouveau, soit d'incorporer celles-ci dans des structures

161 En décembre 2015, le Conseil de paix et sécurité de l'Union africaine avait autorisé l'envoi d'une mission de maintien de la paix de 5000 troupes mais le gouvernement burundais s'y était opposé. En juin 2016, le gouvernement s'opposait à l'envoi, cette fois-ci par les Nations unies, de 228 policiers conformément à la Résolution 2303 (s/2016/659) du Conseil de Sécurité de l'ONU).

162 L'Afrique du Sud justifie son retrait par la tension entre ses obligations en vertu du Statut de Rome et ses obligations internationales au regard du droit international sur les immunités diplomatiques de même que les interventions controversées de la CPI dans des cas impliquant des processus de résolution pacifique des conflits en Afrique. La décision de 1'Afrique du Sud intervient en réaction contre des procédures judiciaires internes à la suite de la visite du président soudanais Omar Hassan $\mathrm{Al}$ Bashir en Afrique du Sud en juin 2015. Depuis mars 2005, Omar Beshir fait l'objet d'un mandat d'arrêt international de la CPI pour crimes de génocide et crimes de guerre qui auraient été commis au Darfour (ICC/02/2005). Sur le sujet, voir Chernor Jalloh «International Justice, Reconciliation and Peace in Africa »(2015) 1 CODESRIA Policy Briefs, en ligne: SSRN <www.papers.ssrn.com> [Jalloh].

163 A l'exception de la situation en Géorgie et si on met de côté les situations sous examen préliminaire, toutes les affaires de même que les situations sous enquête visent des pays africains. Pour plus d'analyse, voir, par exemple Abass, « Problematical Aspects », supra note 84 à la p 47, qui conclut que la CPI a pratiquement besoin de l'Afrique pour survivre.

164 Jalloh, supra note 162 à la p 5.

165 Voir en ce sens Heller, "Radical Complementarity », supra note 129 à la p 5.

166 Burke-White, «Regionalization of International Criminal Law Enforcement», supra note 9 aux pp 748-753. 
judiciaires déjà existantes à l'échelle continentale ${ }^{167}$, comme la manifestation la plus forte du régionalisme. Il estimait par ailleurs que, même si le Statut de Rome était déjà entré en vigueur au moment de l'analyse et que la CPI bénéficiait, à l'époque, d'un soutien considérable de la part des États [c'est nous qui précisons], il ne serait pas inopportun d'explorer cette piste ${ }^{168}$. Burke-White hésitait, il est vrai, à privilégier cette approche de mise en œuvre régionale du droit pénal international par rapport à d'autres modalités envisagées ${ }^{169}$. On peut comprendre les hésitations de cet auteur dans la mesure où il écrivait, comme il le mentionne, durant les premières années d'opérationnalisation de la CPI.

Ces hésitations seront peut-être moins prononcées avec le bilan actuel de la CPI en matière de lutte contre l'impunité de par le monde, maintenant que la Cour est fonctionnelle depuis plus d'une décennie. D'aucuns, parmi les promoteurs de la lutte contre l'impunité, avaient, en effet, espéré que l'avènement d'une cour pénale internationale sonnerait le glas de l'impunité des crimes les plus graves ${ }^{170}$ et rendrait du même coup inopportun le recours à des juridictions de droit pénal international à compétence régionale ou ad hoc. Cependant, la lutte contre l'impunité des crimes les plus graves, en particulier en Afrique, continue de poser des défis qui imposent le recours à ce genre de mécanisme parallèlement à la $\mathrm{CPI}^{171}$.

Sur le plan juridique, une des objections qui pourraient être avancées à l'endroit de la proposition de cet article consisterait dans les risques supposés d'une fragmentation du droit pénal international, laquelle résulterait d'une application régionaliste du droit. À ce sujet, il faut admettre d'emblée que la diversification et l'expansion du droit international ${ }^{172}$ rend ce phénomène inévitable. Cela étant, la

167 La Cour de justice de l'Union européenne ainsi que la Cour européenne des droits de l'homme sur le continent européen, et la Cour-interaméricaine des droits de l'homme au niveau des Amériques. Ibid aux pp 749-750.

168 Ibid à la $\mathrm{p} 749$.

169 Les options sont la CPI siégeant occasionnellement au niveau régional en application de l'article 3 du Statut de Rome, l'application des règles de compétence universelle prioritairement sur une base régionale ou des juridictions nationales spécialisées au sein desquelles siègeraient des juges recrutés sur une base régionale. Ibid aux pp 750-755.

170 L'ancien Secrétaire-Général des Nations Unies, Koffi Annan déclarait, par exemple, ainsi « [i]mpunity has been dealt a decisive blow ». Voir Bureau du Secrétaire-Général, Communiqué de Presse, «Transcriptions d'une conférence de Presse par vidéoconférence entre le Président italien Carlo Ciampi avec le Secrétaire-Général Koffi Annan (Rome et Italie) », UN. Doc. SG/SM/8194 (11 avril 2002) (à la suite de la ratification du Statut de Rome de la Cour Pénale Internationale en Italie), cité dans Burke-White, « Proactive Complementarity », supra note 57 aux pp 53-54

171 En ce sens, Sirleaf note le fait que la création de juridictions de droit pénal international dans les zones affectées par les conflits comme au Soudan du Sud et même en République centrafricaine où la CPI envisage déjà des poursuites reste une option envisagée par les acteurs internationaux dont l'UA et l'ONU. Voir Sirleaf, supra note 8 aux pp 744-745.

172 L'expression vient de la Commission du droit international des Nations unies. Voir ONU, La Commission du droit international et son ceuvre, vol 1, $7^{\mathrm{e}}$ éd, New York, ONU, 2009 à la p 267. Pour des travaux sur ce sujet, voir généralement Carsten Stahn et Larissa van den Herik, "'Fragmentation', Diversification and '3D' Legal Pluralism: International Criminal Law as the Jack-in-the-Box? » dans Larissa van den Herik et Carsten Stahn, dir, The Diversification and Fragmentation of International Criminal Law, The Hague, Grotius Centre for International Legal Studies, 2012, 21 aux pp 21, 75 [Stahn et Herik]. Voir aussi Pierre-Marie Dupuy, « Un débat doctrinal à l'ère de la globalisation : sur la fragmentation du Droit international public » (2000) 1:1 Eur JL Stud aux pp 1-19; Mario Prost, Les 
fragmentation du droit pénal international n'est pas nécessairement préjudiciable. Tout dépend de l'aspect du droit international touché. Si on envisage cette fragmentation du point de vue du droit matériel ou substantiel ${ }^{173}$, plus spécifiquement un risque d'interprétation contradictoire des éléments de qualification des crimes déjà bien établis en droit international, le risque est en réalité minime. En effet, des études comparées de jurisprudence internationale montrent clairement que la prolifération des juridictions de droit international en général ${ }^{174}$, et de droit pénal international en particulier, modifie très peu la substance même de la jurisprudence internationale ${ }^{175}$. Or, c'est justement cet aspect de la fragmentation dont on aurait des raisons fondées de craindre les effets ${ }^{176}$. En revanche, une fragmentation affectant les autres aspects du droit pénal international ${ }^{177}$ n'emporte pas tellement de danger. Elle peut même constituer un enrichissement utile du droit et de la jurisprudence pénale internationale ${ }^{178}$.

$* * *$

Dans les contextes post-conflits comme celui du Burundi, l'application du droit pénal international par un organe judiciaire régional peut permettre d'atteindre des objectifs importants de la justice pénale internationale, difficilement réalisables dans un cadre plus global d'administration de la justice pénale internationale. En raison de sa proximité géographique avec le territoire du pays et des communautés affectées par les crimes, un mécanisme judiciaire régional offre un plus grand potentiel de contribution à la réconciliation ainsi qu'au renforcement des capacités locales des institutions locales dans la lutte contre l'impunité. Fort de ces idées, l'article propose la mise en place d'un organe de droit pénal international au sein de l'EAC. Dans le cas du Burundi, cette option pourrait constituer une alternative viable aux options internes initialement prévues dans le cadre d'un processus interne actuellement dans l'impasse. Le mécanisme judiciaire régional contribuerait également dans la lutte contre l'impunité dans la plupart des États membres de l'EAC en situation post-conflit et qui éprouvent des défis comparables en matière d'État de droit et de lutte contre l'impunité.

unités du droit international et la politique de la fragmentation, thèse de doctorat en droit, Université McGill, 2008 [non publiée]; Anne-Charlotte Martineau, Une analyse critique du débat sur la fragmentation du droit international, thèse de doctorat en droit, Université Panthéon-Sorbonne Paris I, 2013 [non publiée].

173 Voir par ex Stahn et Herik, supra note 172 aux pp 21, 75.

174 Jonathan I Charney, « Is International Law Threatened by Multiple International Tribunals? » (1998) $271 \mathrm{Rec}$ des Cours 101 à la p 125.

175 À propos des tribunaux ad hoc, voir Burke-White, « Regionalization of International Criminal Law Enforcement », supra note 9 à la p 757.

176 Ibid. William W Burke-White souligne les dangers qu'emporterait une modification régionale des éléments du crime contre l'humanité, par exemple.

177 Ibid aux pp 758 et s.

178 Ibid. 
L'article tient compte des développements en cours en Afrique vers la création d'un organe de droit pénal international au sein des structures de l'UA tout en encourageant en même temps l'implication des mécanismes d'intégrations régionales dans la répression des crimes de droit pénal international. Afin de régler les conflits de compétence entre des juridictions supranationales de droit pénal international ainsi envisageables, l'article propose que la plus grande proximité géographique avec le territoire du pays où les crimes ont été commis serve de critère pour déterminer la juridiction supranationale qui devrait bénéficier d'une priorité de compétence pour intervenir, à défaut de poursuite nationale. Cette solution offre le plus grand potentiel de réalisation des objectifs de la justice pénale internationale dans le cadre d'une politique de complémentarité positive. 